\title{
Analytical modeling of the stick-slip motion of an oscillation drum
}

Received: 8 October 2018 / Revised: 8 April 2019 / Published online: 2 July 2019

(C) The Author(s) 2019

\begin{abstract}
In this paper, a lumped parameter model of the interacting oscillation roller-subsurface system is proposed. The main aim of the model is to predict the response acceleration of the roller drum during nearsurface compaction of non-cohesive soils. The compaction process of the soil itself is not captured, but different degrees of compaction are considered by varying the soil stiffness. The roller is represented by the oscillation drum and its viscoelastic connection to the roller frame. In the chosen modeling strategy, the curvature of the soil surface below the drum is prescribed. In this way, also the vertical drum acceleration can be computed. The discrete subsoil model consists of a vertical and a horizontal Kelvin-Voigt element. Contact between drum and soil surface is described by means of dry friction according to Coulomb's law. As such, the stick-slip motion of the drum can be simulated. In the stick phase, pure rolling between drum and soil surface is assumed. The highly nonlinear equations of motion of this three degrees-of-freedom model are derived separately for the stick and the slip phase of the motion. Selective numerical studies show that this model captures the fundamental response characteristics of the dynamic drum-subsoil interacting system observed in the field.
\end{abstract}

\section{Introduction}

A roller, also often referred to as roller-compactor, is a heavy equipment used for near-surface compaction of soil and asphalt layers in the construction of various civil structures such as roads, airfields, dams, and track foundations. A static roller uses only its weight to compact the layer, whereas a dynamic roller enhances the efficiency of subsurface compaction through dynamic excitation of the drum. Depending on the drum excitation, two basic types of dynamic rollers do exist, i.e., vibratory rollers and oscillation rollers. They differ in design, mode of operation, and how the medium to be compacted is loaded. In a vibrating drum, a single unbalance mass, which is attached concentrically to the drum axis, generates a rapidly alternating upwarddownward motion of the drum. The subgrade is compacted by the dynamic pressure applied by the drum. An oscillation drum is equipped with two offset eccentric masses, which rotate synchronously in the same direction. The resulting alternating high-frequency forward-backward motion of the drum, superposed with the translational roller motion, imposes dynamic shear forces that increase the subsurface density.

Continuous Compaction Control (CCC) $([1,11])$ has become the standard technology for controlling subsurface compaction by vibratory rollers. This control technique is based on the dynamic response of the interacting drum-subsurface system recorded during the roller pass, and thus, allows an instant continuous assessment of the degree of compaction. For oscillation rollers, however, no mature CCC system has been

I. Paulmichl · C. Adam ( $₫)$

Unit of Applied Mechanics, University of Innsbruck, 6020 Innsbruck, Austria

E-mail: christoph.adam@uibk.ac.at

D. Adam

Institute of Geotechnics, TU Wien, 1040 Vienna, Austria 
developed yet. Based on the data of field tests with an oscillation roller, recently, it was discovered that the area enclosed in the figure which is formed when plotting the vertical against the horizontal acceleration of the drum center, is a characteristic quantity for the compaction degree of the subsurface [19]. Since, however, experiments provide only a selective insight into the response behavior of the entire parameter space, a complete picture of the drum response and its parameter interdependency can only be gained by the outcomes of comprehensive parametric numerical studies.

The literature on mechanical modeling of roller-compactors for numerical response simulation is quite scarce. Basically, it can be distinguished between finite element (FE) models (e.g., [3,9]) and lumped parameter models (e.g., $[2,14,23])$ of the dynamic interacting roller-subsurface system. The focus of most FE models is to predict the subsurface compaction and, depending on the degree of sophistication, allow only selective insight into the system response, like experimental studies. In contrary, lumped parameter models are often used to predict the response of the compaction device for predefined subsurface condition or (over)simplified soil compaction models.

A first modeling attempt of the oscillation drum-soil system was presented in [27], where a single degreeof-freedom (SDOF) model captures slip between drum and subsurface, considering the suspension elements between drum and frame. This paper also explains the working principle of oscillation compaction and some basic response phenomena such as "peak cut" of the horizontal drum acceleration. In a similar approach, Kopf [11] studied the influence of the slip motion on the horizontal drum accelerations, using a lumped parameter model with a SDOF. More recently, Pistrol [18] presented a three degrees-of-freedom (3DOF) model for the drum in pure rolling motion that delivers the horizontal and also the vertical acceleration response of the drum. Lately, in [14] the amplitude frequency characteristics of a tandem oscillation roller have been studied based on a 5DOF model in pure rolling motion. From this brief literature survey, it can be concluded that, so far, no reliable mechanical model that predicts both realistically and efficiently the dynamic response of an oscillation drum is available.

To fill this gap, in the present contribution a lumped parameter model is developed that facilitates the response simulation of an oscillation drum with the least numerical effort, and captures the observed stick-slip motion of the drum during soil compaction. In the chosen strategy, a curved dent, which approximates the settlement trough of the subsoil below the drum, is prescribed, supported by a discrete spring-damper soil model [18]. This curved dent allows to simulate the vertical motion of the drum. The drum is modeled as a circular rigid body with spring-damper elements attached to its center, which represent the viscoelastic connection to the roller frame. In addition to the sinusoidal excitation torque induced by the unbalance masses, a constant driving torque is imposed to the drum. As such, the effect of the translational motion of the roller on the response can be captured. It is, however, not the aim to simulate the compaction process itself but the soil parameters are a priori defined.

The paper is organized as follows. After a brief explanation of the basic components and the working principle of an oscillation roller, the proposed drum-subsoil interacting model and excitation model are presented. Based on the kinematic relations, in a substructure approach the equations of motion of this 3DOF model are derived separately for the stick and the slip motion phase, and the numerical solution of these highly nonlinear equations is discussed. Once the model has been established, the impact of the contact condition between drum and soil (pure rolling vs. stick-slip motion), soil stiffness, and driving torque on the response of the oscillation drum of a specific roller is investigated in detail. The obtained results allow to detect, to understand, and to explain better the dynamic response of the interacting oscillation drum-soil system, and ultimately to validate CCC techniques for oscillation rollers.

\section{Mechanical model}

\subsection{Basic structure and mode of operation of an oscillation roller}

The principal components of an oscillation roller shown in Fig. 1 are the rigid drum (at the front) equipped with two rotating unbalances (also referred to as unbalanced masses or eccentric masses), the driving wheels, the front and the rear frame, and the driver's cabin including processing, display, and storing unit. Drum and front frame are separated by suspension elements (rubber buffers) to prevent the transmission of vibrations from the drum to the frame. A hinged connection between the front frame and the rear frame improves the maneuverability of the roller. To protect the driver from vibrations, the cabin is detached from the rear frame by isolation mounts. 


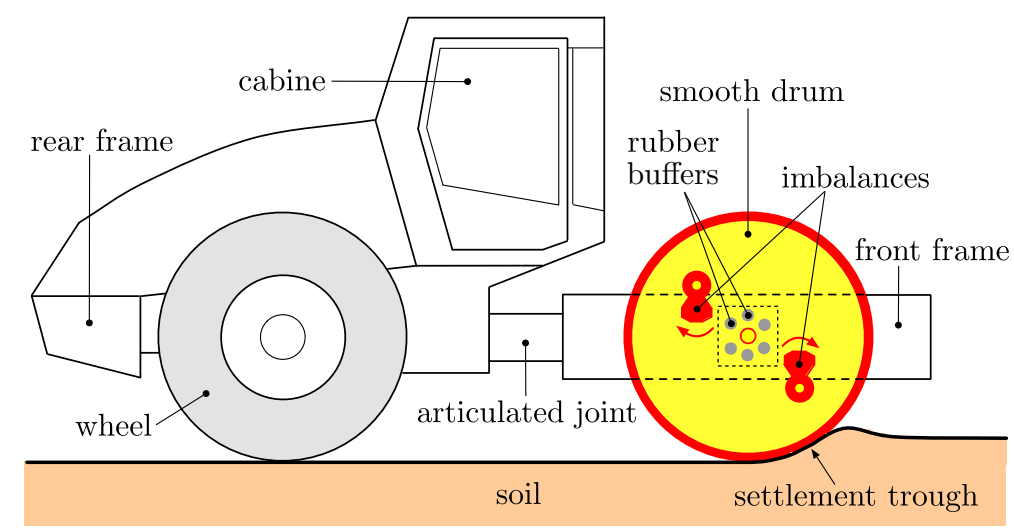

Fig. 1 Principal components of an oscillation roller-compactor with smooth drum

In the oscillation drum, two opposite eccentric masses, whose shafts are arranged excentrically to the drum axis, rotate synchronously in the same direction (see Fig. 1), driven by a toothed belt. Because these unbalance masses are offset by $180^{\circ}$ relative to each other, the out-of-balance forces cancel out. The remaining spinning couple of forces induces a torsional moment around the drum axis, which changes its sign during the rotation of the eccentric masses. It causes the drum to rapidly move in an alternating forward-backward motion. This rotational motion and the translational motion of the roller moving with constant speed are superposed. The dynamic (alternating) tangential (shear) forces, imposed through friction in the contact area between drum and surface of the compacted medium, induce mainly shear waves in the subsurface, and compaction is achieved by "massaging" the material [8], also referred to as shear force compaction [22]. Unlike in a vibrating drum, which may bounce during compaction $[2,23]$, the compaction forces strain continuously the subsoil because oscillation drum and soil remain in permanent contact. An oscillation drum performs either a pure rolling motion or a stick-slip motion. As such, an oscillation roller continuously compacts the subsurface both dynamically by alternating (primarily) horizontal shear loading and statically by its weight in the vertical direction. During the forward motion, the static weight of the drum deforms the soil surface asymmetrically as shown in Fig. 1, subsequently referred to as "settlement trough". Since the contact zone between the drum and subsoil is curved, the oscillation drum responds also in the vertical direction.

\subsection{Representation of roller and subsoil}

The main objective of the roller-soil model to be developed is to allow for a comprehensive parametric study of the response behavior of the drum for a given subsoil condition (i.e., the compaction process in the soil is not simulated). Consequently, in the desired computationally efficient model all parts of the roller with minor effects on the dynamic drum response are omitted. During operation, both the horizontal and vertical vibrations of the rear and front frames are negligible because drum and front frame are dynamically decoupled by the deeply tuned rubber buffers. For further details, see Appendix A. Based on this observation, it is reasonable to reduce the whole roller to the oscillation drum that is connected through spring-damper elements to the static frame, taking into account the dead weight of the front frame. The drum is assumed to be a planar rigid smooth circular body of radius $r$ with mass $m$ and mass moment of inertia $I$ (with respect to drum center $M$ ). Vertical and horizontal spring-damper elements attached to the drum center, both of same stiffness $\left(k_{\mathrm{d}}\right)$ and viscous damping $\left(c_{\mathrm{d}}\right)$ properties, capture the effect of the rubber buffers, as shown in Fig. 2.

In the utilized modeling strategy, the shape of the soil surface below the drum, which has a significant impact on the roller response, and the soil parameters depending on the degree of soil compaction need to be defined a priori (see, for instance, $[11,18]$ ). The settlement trough is prescribed as an asymmetric rigid curved track according to a logarithmic spiral. At the bottom of the settlement trough (point $A$ in Fig. 2), in a common approach the effect of the elastic continuous halfspace (subsoil) is captured simplified through two discrete Kelvin-Voigt bodies ([2,23,25]), one arranged in vertical direction (subscript $s v$ ) and one in horizontal (subscript sh) direction. The settlement trough exhibits a translational motion in both horizontal and vertical directions; its rotation is, however, constrained. In [5] and [25], the reduction in the elastic soil halfspace to spring-dashpot damper elements in parallel is discussed in detail, and thus, not repeated here. The expressions 


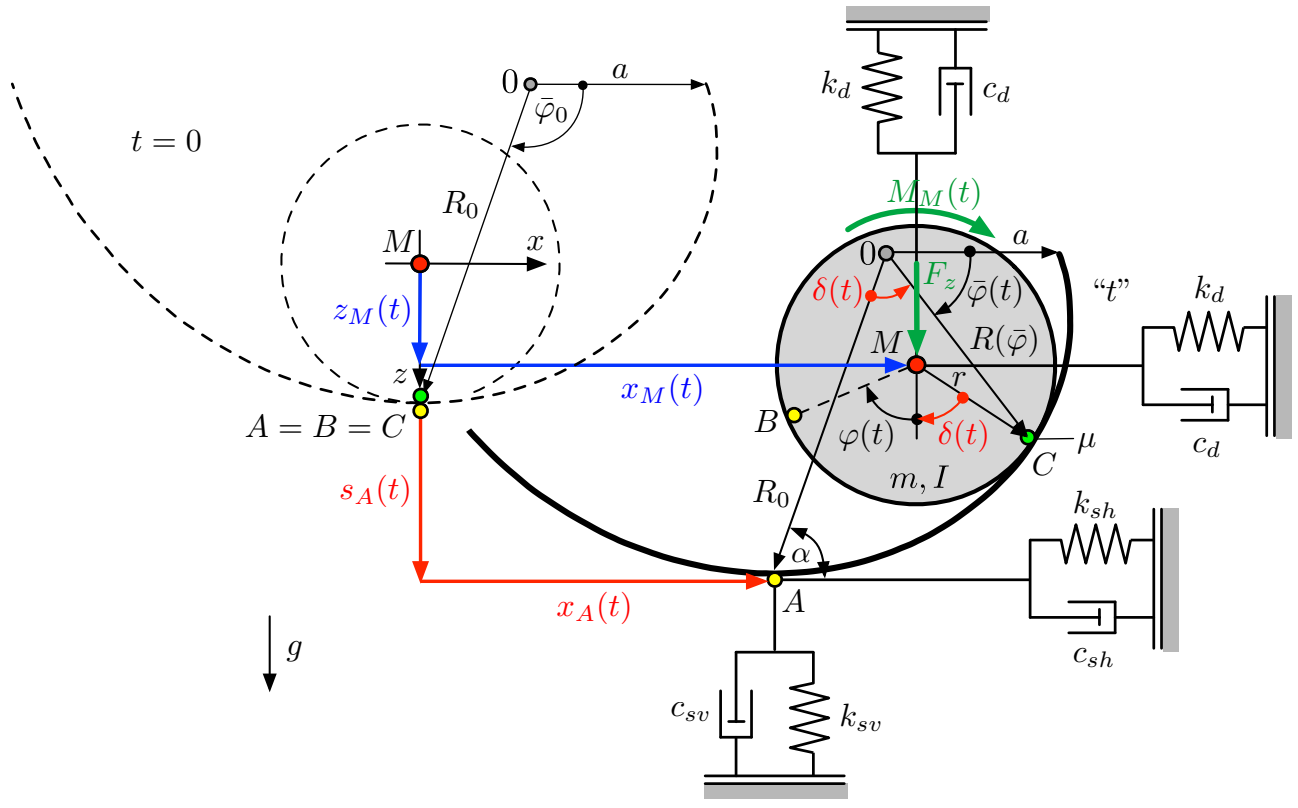

Fig. 2 Mechanical model of the interaction system oscillation roller-soil

for the soil spring coefficients $k_{\mathrm{sh}}$ and $k_{\mathrm{sv}}$ and the soil damping parameters $c_{\mathrm{Sh}}$ and $c_{\mathrm{sv}}$ are listed in Appendix B. In the current roller-soil model, the soil mass is not considered because its effect is negligible if Poisson's ratio $v$ of the soil is less than $\frac{1}{3}$ [25]. This is the case in the current study where only non-cohesive soils are considered. For larger Poisson's ratio, a trapped soil mass is added to the vertical spring-dashpot damper elements, as explained in [25].

The contact between drum and subsoil is described by means of dry friction according to Coulomb's law with constant coefficient of friction $\mu$. As such, both the stick phase and the slip phase between drum and soil can be simulated by the roller-soil interaction model shown in Fig. 2, which has 3DOF.

\subsection{Modeling of excitation and loading}

The two rotating eccentric shafts inside the drum with each two imbalances (see Fig. 1) can be each represented by two equal eccentric lumped masses, $m_{u 1}=m_{u 2}=2 m_{u}$, with distance $e_{u}$ from the center of rotation (see Fig. 3). The amplitude $F_{u}$ of the centripetal force generated by each unbalanced shaft rotating with constant angular velocity $\bar{v}$ is

$$
F_{u 1}=F_{u 2}=F_{u}=2 m_{u} e_{u} \bar{v}^{2}
$$

where $\bar{v}$ is $2 \pi$ times the excitation frequency $\bar{f}$. Decomposition of the centripetal force at time $t$ into component $F_{u \|}$ in parallel and component $F_{u \perp}$ perpendicular to the line that connects the center of rotation (denoted as $R C$ in Fig. 3) and the drum center $M$ yields

$$
F_{u \|}=F_{u} \cos (\bar{v} t), \quad F_{u \perp}=F_{u} \sin (\bar{v} t)
$$

Since the unbalance masses $m_{u 1}$ and $m_{u 2}$ are offset by $180^{\circ}$ relative to each other and the shafts rotate in the same direction, the components of the corresponding unbalance forces

$$
\begin{gathered}
\vec{F}_{u \| 1}=-F_{u \|} \vec{e}_{\|}, \quad \vec{F}_{u \perp 1}=-F_{u \perp} \vec{e}_{\perp} \\
\vec{F}_{u \| 2}=F_{u, \|} \vec{e}_{\|}, \quad \vec{F}_{u \perp 2}=F_{u \perp} \vec{e}_{\perp}
\end{gathered}
$$

cancel out. 

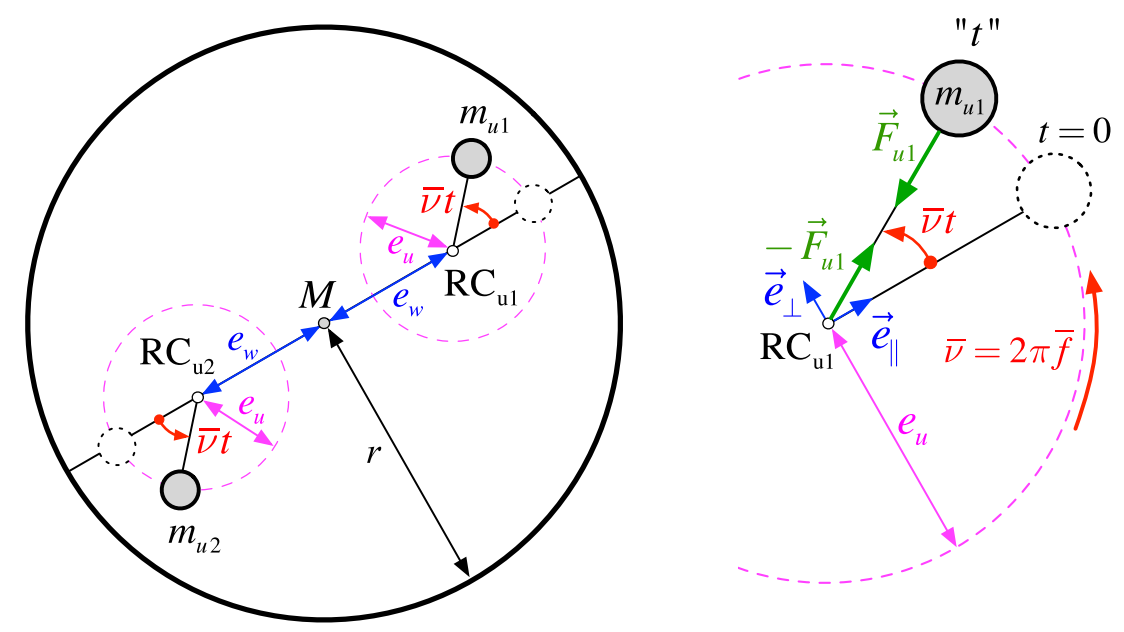

Fig. 3 Drum with unbalanced shafts (left), and circular motion of one unbalance mass with constant circular frequency $\bar{v}$ (right)

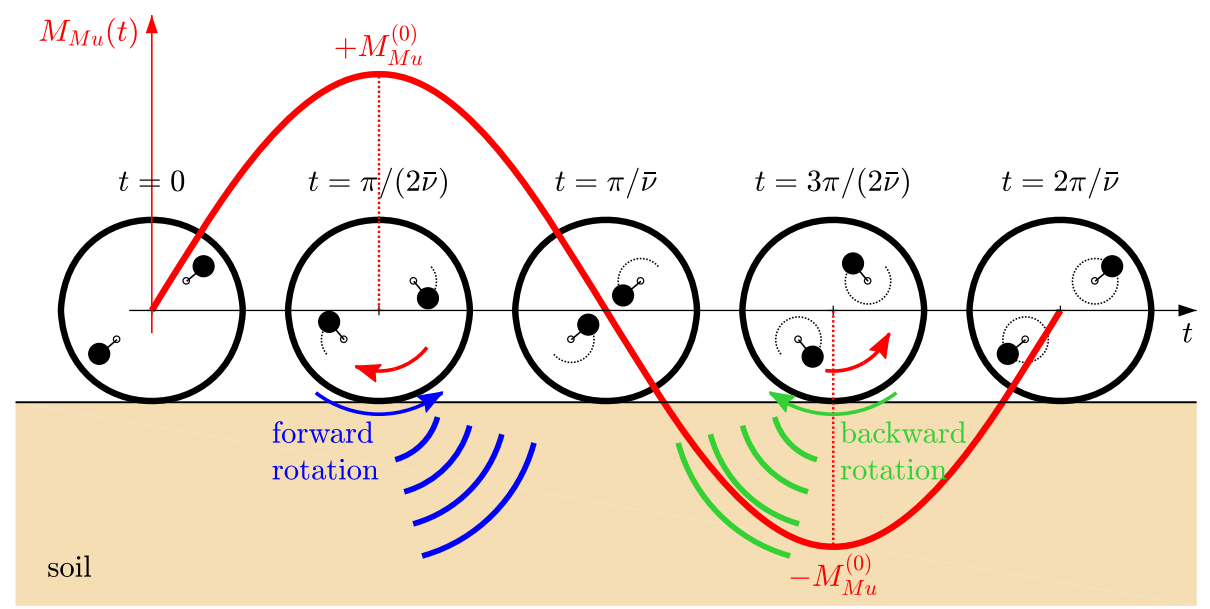

Fig. 4 Excitation torque $M_{\mathrm{Mu}}(t)$ in an oscillation drum and corresponding location of the unbalance at discrete time instants, based on [27]

In the above equations, $\vec{e}_{\|}$and $\vec{e}_{\perp}$ denote the unit vectors depicted in Fig. 3. The counteracting normal forces $\vec{F}_{u \perp 1}$ and $\vec{F}_{u \perp 2}$ with distance $2 e_{w}$ result in the sinusoidal torque $M_{\mathrm{Mu}}(t)$ around the drum axis with the amplitude $M_{\mathrm{Mu}}^{(0)}$,

$$
M_{\mathrm{Mu}}(t)=M_{\mathrm{Mu}}^{(0)} \sin (\bar{v} t), \quad M_{\mathrm{Mu}}^{(0)}=4 m_{u} e_{u} e_{w} \bar{v}^{2} .
$$

In Fig. 4, the relation between the excitation torque $M_{\mathrm{Mu}}(t)$ and the location of the rotating unbalanced shafts is visualized.

The total drum excitation applied at the drum center is composed of the unbalanced shaft moment $M_{\mathrm{Mu}}(t)$ and the constant driving torque $M_{\mathrm{Md}}$ imposed by the roller engine,

$$
M_{M}(t)=M_{\mathrm{Mu}}(t)+M_{\mathrm{Md}}
$$

Application of the constant torque $M_{\mathrm{Md}}$ allows controlling the location of the drum in the settlement trough. Since the oscillation drum motion is superposed to the translational motion of the drum with constant roller velocity, the drum does not oscillate around the bottom of the settlement trough but on its slope in the driving direction. In addition, the vertical load $F_{z}$, i.e., the static axle load $P_{0}$ of the frame minus drum weight $m g$,

$$
F_{z}=P_{0}-m g
$$

is applied to the drum center. The variable $g$ denotes the acceleration of gravity. 
In contrast to a simpler roller model presented in [18], the model proposed here can be used to simulate the stick-slip motion of the drum, to take into account the suspension between drum and roller, to consider the effect of driving velocity by application of a constant driving torque, and to study the motion of the oscillation drum in its asymmetric settlement trough.

\section{Governing equations}

\subsection{Kinematics}

Before the equations of motion are derived, the kinematic relations for the drum motion and soil-drum interaction need to be established.

The distance $R$ from the origin of the logarithmic spiral (point 0 ) describing the settlement trough to the contact point $C$ at time $t$ reads as [24]

$$
R(t)=a \exp [k \bar{\varphi}(t)], \quad \bar{\varphi}(t)=\bar{\varphi}_{0}-\delta(t),
$$

with the spiral parameters $a$ and $k$ calibrated to the actual soil surface shape. The angle $\bar{\varphi}_{0}=\pi-\alpha$ represents the inclination of the connection line between 0 and $C$ at time $t=0, \alpha=\arctan \frac{1}{k}$ is the angle between the tangent and radial line at point $\left(R_{0}, \bar{\varphi}_{0}\right)$, and $\delta(t)$ defines the position of the drum in the settlement trough at time $t$ (see Fig. 2). It is assumed that at time $t=0$, the drum is at the bottom of the rigid settlement trough, as indicated by dashed lines in Fig. 2 . This position $\left(R_{0}, \bar{\varphi}_{0}\right)$ is defined by the radius $R_{0}=a \exp \left(k \bar{\varphi}_{0}\right)$ and angle $\bar{\varphi}_{0}$. For $k \rightarrow 0, \alpha \rightarrow \pi / 2$, the spiral approaches a circle with radius $a$. Thus, with the proposed model both a symmetric semicircular and an asymmetric spiral-shaped settlement trough can be captured.

In the stick phase of the drum, the following three independent coordinates are chosen to describe the motion of 3DOF model shown in Fig. 2, i.e., the displacement components $x_{A}(t)$ and $s_{A}(t)$ of support point $A$, and the position angle $\delta(t)$. In this phase, continuous rolling contact can be assumed, and the relative velocity between drum surface and settlement trough is zero. Consequently, the arc length $L_{A C}$ along the settlement trough (between support point $A$ and the contact point $C$, see Fig. 2),

$$
L_{A C}=\frac{\sqrt{1+k^{2}}}{k} R_{0}[1-\exp (-k \delta)]
$$

and the arc length $L_{B C}$ along the drum surface (between contact point $C$ and point $B$ on the drum, see Fig. 2),

$$
L_{B C}=(\varphi+\delta) r
$$

are equal, $L_{A C}=L_{B C}$. In Eq. 10, $\varphi(t)$ denotes the total rotation angle of the drum [15]. Rewriting this rolling condition yields for the stick phase the relationship between the drum rotation angle $\varphi$ and the position angle $\delta$

$$
\varphi=\frac{\sqrt{1+k^{2}}}{k r} R_{0}[1-\exp (-k \delta)]-\delta
$$

The first and the second time derivative read

$$
\dot{\varphi}=f_{5} \dot{\delta}, \quad \ddot{\varphi}=f_{5} \ddot{\delta}+f_{6} \dot{\delta}^{2} .
$$

The functions $f_{5}$ and $f_{6}$, which depend on the angle $\delta(t)$ and the spiral parameters $R_{0}$ and $k$, are listed in Appendix C.

Slip of the drum on the soil surface results in a relative motion between the drum and soil. Thus, in the slip phase the lengths $L_{A C}$ (Eq. 9) and $L_{B C}$ (Eq. 10) are dissimilar. Differentiation of the difference $L_{A C}-L_{B C}$ with respect to time $t$ results in the relative velocity $v_{\text {rel }}$ between the drum and subsoil (slip velocity),

$$
v_{\text {rel }}=\dot{\delta} f_{5} r-\dot{\varphi} r
$$

Since in the slip phase $v_{\text {rel }} \neq 0$, the angles $\delta(t)$ and $\varphi(t)$ become independent variables. However, the tangential friction contact according to Coulomb's law at point $C$ couples in this phase the variables $x_{A}$ and $s_{A}$, which are, therefore, no independent variables, as is seen later. 
For deriving the equations of motion, it is convenient to express the horizontal and vertical displacement components $x_{M}$ and $z_{M}$, respectively, of the drum center $M$ at time $t$ as a function of the coordinates $x_{A}(t), s_{A}(t)$, and $\delta(t)$,

$$
\begin{aligned}
x_{M} & =x_{M}^{(\text {roll })}+x_{A}, \quad z_{M}=z_{M}^{(\text {roll })}+s_{A}, \\
x_{M}^{(\text {roll })} & =-R_{0} \exp (-k \delta) \cos (\alpha+\delta)-r \sin \delta+R_{0} \cos \alpha, \\
z_{M}^{(\text {roll })} & =R_{0} \exp (-k \delta) \sin (\alpha+\delta)-r \cos \delta-\left(R_{0} \sin \alpha-r\right),
\end{aligned}
$$

where $x_{M}^{(\text {roll) }}$ and $z_{M}^{(\text {roll })}$ are the corresponding displacement components of $M$ relative to the settlement trough, which are superposed to the displacement components of the settlement trough, $x_{A}$ and $s_{A}$, respectively. Differentiation of Eq. (14) with respect to time $t$ yields the components of the drum center velocity,

$$
\dot{x}_{M}=f_{1} \dot{\delta}+\dot{x}_{A}, \quad \dot{z}_{M}=f_{3} \dot{\delta}+\dot{s}_{A},
$$

and repeated differentiation the acceleration components,

$$
\ddot{x}_{M}=f_{1} \ddot{\delta}+f_{2} \dot{\delta}^{2}+\ddot{x}_{A}, \quad \ddot{z}_{M}=f_{3} \ddot{\delta}+f_{4} \dot{\delta}^{2}+\ddot{s}_{A}
$$

in terms of $x_{A}(t), s_{A}(t)$, and $\delta(t)$. The functions $f_{1}, f_{2}, f_{3}, f_{4}, f_{5}, f_{6}$, which depend on the angle $\delta(t)$, the spiral parameters $a, k, \alpha, R_{0}$ and the drum radius $r$, are specified in "Appendix C."

\subsection{Dynamic substructuring}

To derive efficiently the equations of motion, the model of Fig. 2 is separated into the subsystem drum $(I)$ and the subsystem soil (II) including the settlement trough. At the contact point $C$, the normal component $N$ and the tangential component $T$ of the interface force are applied as external forces, as shown in Figs. 5 and 6.

\section{Subsystem I: Drum}

Two equations are obtained by the application of conservation of momentum [26] to the subsystem drum in horizontal $(x)$ and vertical $(z)$ directions, compared with Fig. 5,

$$
\begin{aligned}
& T \cos \delta-N \sin \delta-c_{\mathrm{d}} \dot{x}_{M}-k_{\mathrm{d}} x_{M}=m \ddot{x}_{M}, \\
& \left(m g+F_{z}\right)-T \sin \delta-N \cos \delta-c_{\mathrm{d}} \dot{z}_{M}-k_{\mathrm{d}} z_{M}=m \ddot{z}_{M} .
\end{aligned}
$$

Solving this coupled system of equations for $N$ and $T$ yields

$$
\begin{aligned}
N & =\left[\left(m g+F_{z}\right)-\left(m \ddot{z}_{M}+c_{\mathrm{d}} \dot{z}_{M}+k_{\mathrm{d}} z_{M}\right)\right] \cos \delta-\left(m \ddot{x}_{M}+c_{\mathrm{d}} \dot{x}_{M}+k_{\mathrm{d}} x_{M}\right) \sin \delta, \\
T & =\left[\left(m g+F_{z}\right)-\left(m \ddot{z}_{M}+c_{\mathrm{d}} \dot{z}_{M}+k_{\mathrm{d}} z_{M}\right)\right] \sin \delta+\left(m \ddot{x}_{M}+c_{\mathrm{d}} \dot{x}_{M}+k_{\mathrm{d}} x_{M}\right) \cos \delta .
\end{aligned}
$$

A third equation is obtained by the application of conservation of angular momentum [26] with respect to the drum center $M$,

$$
I \ddot{\varphi}=M_{M}(t)-T r,
$$

where $\varphi$ denotes the total rotation angle [15] of the drum (see Fig. 5).

\section{Subsystem II: Subsoil}

Conservation of momentum in horizontal $(x)$ and vertical $(z)$ directions to the soil subsystem shown in Fig. 6 leads to

$$
\begin{aligned}
& N \sin \delta-T \cos \delta=k_{\mathrm{Sh}} x_{A}+c_{\mathrm{sh}} \dot{x}_{A}, \\
& N \cos \delta+T \sin \delta=k_{\mathrm{Sv}} s_{A}+c_{\mathrm{Sv}} \dot{s}_{A} .
\end{aligned}
$$

This set of equations is solved for $N$,

$$
N=\left(k_{\mathrm{sh}} x_{A}+c_{\mathrm{sh}} \dot{x}_{A}\right) \sin \delta+\left(k_{\mathrm{sv}} s_{A}+c_{\mathrm{sv}} \dot{s}_{A}\right) \cos \delta .
$$




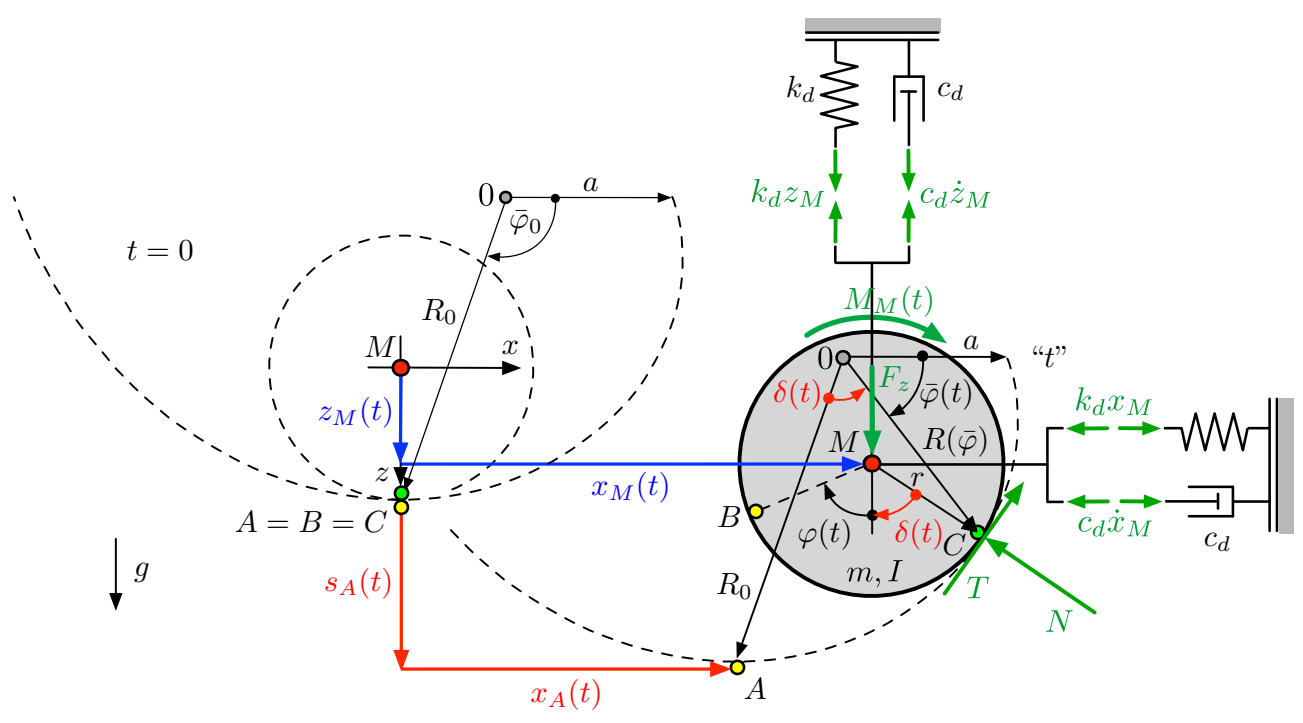

Fig. 5 Subsystem I: oscillation drum with rubber buffers

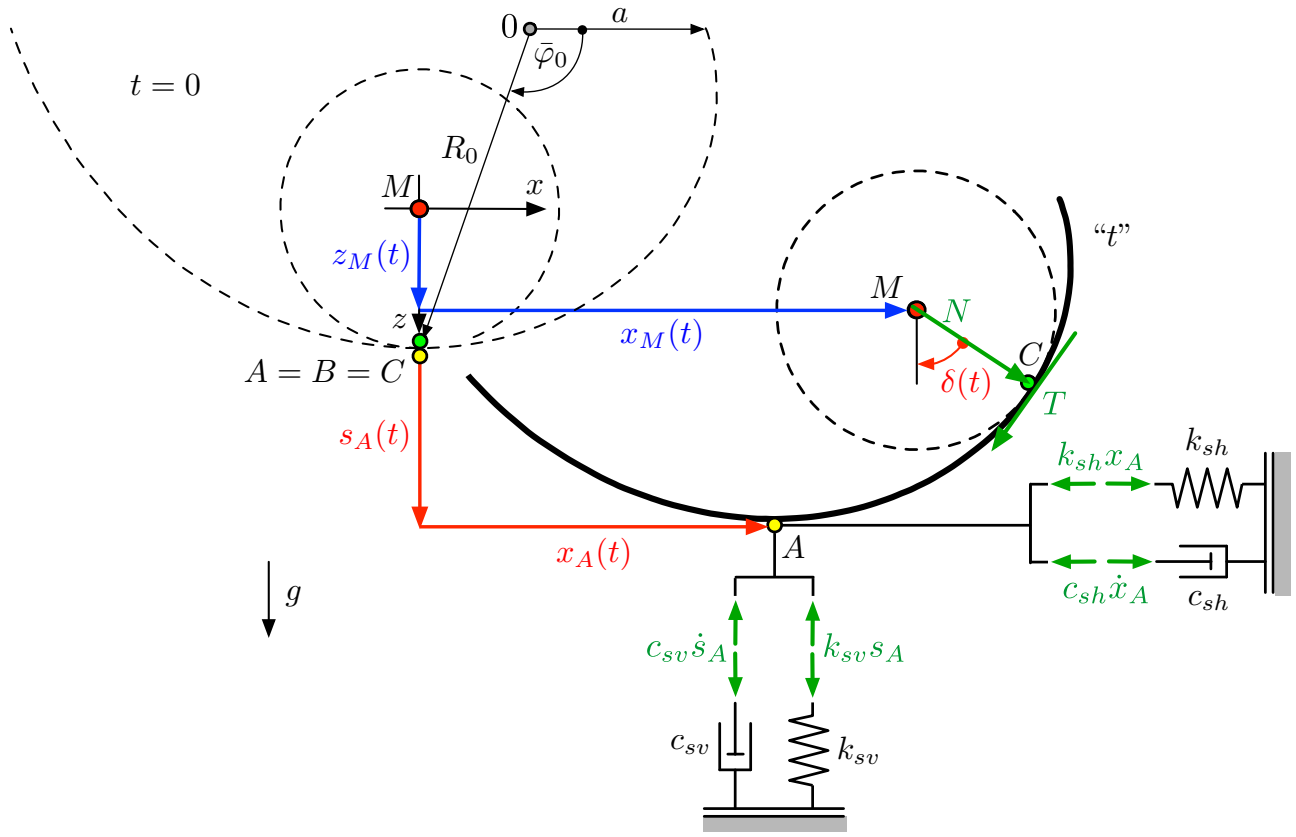

Fig. 6 Subsystem II: subsoil including settlement trough

\section{Coupling of the subsystems}

The compatibility conditions at contact point $C$ between both subsystems depend on the motion phase (stick or slip) of the drum.

In the stick phase, where kinematic relations Eqs. 11 and 12 are applicable, the sliding friction force $T_{\mathrm{f}}$ between drum and soil according to Coulomb's law of dry friction [21]

$$
T_{\mathrm{f}}=-\mu N \operatorname{sign}\left(v_{\mathrm{rel}}\right)
$$

is not exceeded at any time,

$$
|T| \leq\left|T_{\mathrm{f}}\right|=\mu N \quad\left(v_{\text {rel }}=0\right) .
$$

It is assumed that the coefficient of kinetic friction $\mu_{k}$ is approximately equal to the coefficient of static friction $\mu_{s}[20]$, and thus, $\mu=\mu_{k} \approx \mu_{s}$. 
If $|T|=\mu N$ and if there is a relative motion between drum and subsoil with relative velocity $v_{\text {rel }}$ according to Eq. 13, the motion switches from the stick phase to the slip phase. In the slip phase, where $v_{\text {rel }} \neq 0$, the tangential contact force $T$ corresponds to Coulomb's force of friction $T_{\mathrm{f}}$, depending on the sign of $v_{\text {rel }}$,

$$
T=\left|T_{\mathrm{f}}\right|>0 \quad\left(v_{\text {rel }} \leq 0^{-}\right), \quad T=-\left|T_{\mathrm{f}}\right|<0 \quad\left(v_{\text {rel }} \geq 0^{+}\right)
$$

\subsection{Equations of motion}

\section{Stick phase}

Adding up Eqs. (19) and (24), and replacing $x_{M}$ and its first and second derivatives by the independent coordinates $\delta$ and $x_{A}$ and their derivatives through Eqs. (14), (17), and (18), leads to the first equation of motion in terms of $\delta$ and $x_{A}$,

$$
\begin{aligned}
& f_{1} m \ddot{\delta}+m \ddot{x}_{A}+h_{1}=0, \\
& h_{1}=f_{2} m \dot{\delta}^{2}+f_{1} c_{\mathrm{d}} \dot{\delta}+\left(c_{\mathrm{sh}}+c_{\mathrm{d}}\right) \dot{x}_{A}+\left(k_{\mathrm{sh}}+k_{\mathrm{d}}\right) x_{A}+k_{\mathrm{d}} x_{M}^{(\text {roll })} .
\end{aligned}
$$

Similarly, Eqs. (20) and (25) are added up, and $z_{M}$ and its derivatives are expressed by $\delta$ and $s_{A}$ through Eqs. (14), (17) and (18), respectively, yielding the second equation of motion,

$$
\begin{aligned}
& f_{3} m \ddot{\delta}+m \ddot{s}_{A}+h_{2}=m g+F_{z}, \\
& h_{2}=f_{4} m \dot{\delta}^{2}+f_{3} c_{\mathrm{d}} \dot{\delta}+\left(c_{\mathrm{Sv}}+c_{\mathrm{d}}\right) \dot{s}_{A}+\left(k_{\mathrm{Sv}}+k_{\mathrm{d}}\right) s_{A}+k_{\mathrm{d}} z_{M}^{(\text {roll })} .
\end{aligned}
$$

To obtain the third equation of motion in terms of the independent coordinates $\delta, x_{A}$ and $s_{A}$, in Eq. (23), $T$ is substituted with Eq. (22) and $\ddot{\varphi}$ with Eq. (12). Then, $x_{M}$ and $z_{M}$ and their time derivatives are replaced by the relations of Eqs. (14), (17), and (18), resulting after some algebra in

$$
\begin{aligned}
& \left(f_{5} \frac{I}{m r}+\tilde{f}_{1}\right) m \ddot{\delta}+m \ddot{x}_{A} \cos \delta-m \ddot{s}_{A} \sin \delta+h_{3}=\frac{M_{M}(t)}{r}-\left(m g+F_{z}\right) \sin \delta, \\
& h_{3}=\left(f_{6} \frac{I}{m r}+\tilde{f}_{2}\right) m \dot{\delta}^{2}+c_{\mathrm{d}}\left[\tilde{f}_{1} \dot{\delta}+\dot{x}_{A} \cos \delta-\dot{s}_{A} \sin \delta\right] \\
& \quad+k_{\mathrm{d}}\left[\left(x_{M}^{\text {(roll })}+x_{A}\right) \cos \delta-\left(z_{M}^{(\text {roll })}+s_{A}\right) \sin \delta\right],
\end{aligned}
$$

with

$$
\tilde{f}_{1}=f_{1} \cos \delta-f_{3} \sin \delta, \quad \tilde{f}_{2}=f_{2} \cos \delta-f_{4} \sin \delta .
$$

Equations (30), (31), and (32) represent three coupled second-order nonlinear ordinary differential equations (ODEs), which describe the motion of the 3DOF roller-soil interaction model in the stick phase and for pure rolling. For efficient numerical solution, this coupled set of equations is written in the state space, as described in Appendix D.

It should be noted that for an immovable semicircular settlement trough (i.e., $k=0, k_{\mathrm{sh}} \rightarrow \infty$ and $k_{\mathrm{sv}} \rightarrow \infty$ ), Eq. (32) approaches the solution presented in [26] (example A7.2, pp 461-462). On the other hand, if the settlement trough becomes horizontal (i.e., $R \rightarrow \infty$ ), Eq. (32) reduces to the equation of motion of a model proposed in [11].

\section{Slip phase}

To derive the first equation of motion of the 3DOF model in the slip phase, in Eqs. (19) and (20) the tangential force component $T$ is replaced by the expression of Eq. (27), representing the friction force $T_{\mathrm{f}}$. These equations are combined by eliminating the normal force component $N$. Then, $x_{M}$ and $z_{M}$ and their time derivatives are expressed by means of the coordinates $\delta, x_{A}, s_{A}$ and their time derivatives according to Eqs. (14), (17), and (18), leading to

$$
\begin{aligned}
& \left(f_{1} f_{\mu 1}-f_{3} f_{\mu 2}\right) m \ddot{\delta}+f_{\mu 1} m \ddot{x}_{A}-f_{\mu 2} m \ddot{s}_{A}+h_{1}^{(\mathrm{sl})}=-f_{\mu 2}\left(m g+F_{z}\right), \\
& h_{1}^{(\mathrm{sl})}=\left(f_{2} f_{\mu 1}-f_{4} f_{\mu 2}\right) m \dot{\delta}^{2}+c_{\mathrm{d}}\left[\left(f_{1} f_{\mu 1}-f_{3} f_{\mu 2}\right) \dot{\delta}+f_{\mu 1} \dot{x}_{A}-f_{\mu 2} \dot{s}_{A}\right] \\
& \quad+k_{\mathrm{d}}\left[f_{\mu 1}\left(x_{M}^{\text {(roll) }}+x_{A}\right)-f_{\mu 2}\left(z_{M}^{\text {(roll })}+s_{A}\right)\right]
\end{aligned}
$$


with

$$
f_{\mu 1}=\cos \delta-\operatorname{sign}\left(v_{\text {rel }}\right) \mu \sin \delta, \quad f_{\mu 2}=\sin \delta+\operatorname{sign}\left(v_{\text {rel }}\right) \mu \cos \delta
$$

The second equation of motion is based on Eq. (23) derived by conservation of angular momentum, where $T$ is likewise replaced by $T_{\mathrm{f}}$ according to Eq. (27), and $N$ is substituted with Eq. (21). In the resulting relation, $x_{M}$ and $z_{M}$ and their time derivatives are substituted as before, with the outcome

$$
\begin{aligned}
& \operatorname{sign}\left(v_{\text {rel }}\right) \mu m\left(\tilde{f}_{3} \ddot{\delta}+\ddot{x}_{A} \sin \delta+\ddot{s}_{A} \cos \delta\right)+\frac{I}{r} \ddot{\varphi}+h_{2}{ }^{(\mathrm{sl})}=\frac{M_{M}(t)}{r}, \\
& h_{2}{ }^{(\mathrm{sl})}=--\operatorname{sign}\left(v_{\text {rel }}\right) \mu\left\{\left(m g+F_{z}\right) \cos \delta-\tilde{f}_{4} m \dot{\delta}^{2}-\tilde{f}_{3} c_{\mathrm{d}} \dot{\delta}-c_{\mathrm{d}}\left(\sin \delta \dot{x}_{A}+\cos \delta \dot{s}_{A}\right)\right. \\
&\left.-k_{\mathrm{d}}\left[\left(x_{M}^{(\text {roll })}+x_{A}\right) \sin \delta+\left(z_{M}^{(\text {roll })}+s_{A}\right) \cos \delta\right]\right\},
\end{aligned}
$$

where

$$
\tilde{f}_{3}=f_{1} \sin \delta+f_{3} \cos \delta, \quad \tilde{f}_{4}=f_{2} \sin \delta+f_{4} \cos \delta
$$

The third equation of motion results from coupling of the two subsystems. To this end, Eqs. (21) and (26) are combined through $N$. Considering Eq. (37), also this equation of motion is written in terms of the kinematic variables $\delta, x_{A}, s_{A}$, and their time derivatives,

$$
\begin{aligned}
& m \tilde{f}_{3} \ddot{\delta}+m \ddot{x}_{A} \sin \delta+m \ddot{s}_{A} \cos \delta+h_{3}{ }^{(\mathrm{sl})}=\left(m g+F_{z}\right) \cos \delta \\
& h_{3}{ }^{(\mathrm{sl})}= \\
& \quad c_{\mathrm{d}} \tilde{f}_{3} \dot{\delta}+\left(c_{\mathrm{sh}}+c_{\mathrm{d}}\right) \dot{x}_{A} \sin \delta+\left(c_{\mathrm{sv}}+c_{\mathrm{d}}\right) \dot{s}_{A} \cos \delta+m \tilde{f}_{4} \dot{\delta}^{2} \\
& \quad+\left(k_{\mathrm{sh}}+k_{\mathrm{d}}\right) x_{A} \sin \delta+\left(k_{\mathrm{sv}}+k_{\mathrm{d}}\right) s_{A} \cos \delta+k_{\mathrm{d}}\left(x_{M}^{(\text {roll })} \sin \delta+z_{M}^{(\text {roll })} \cos \delta\right) .
\end{aligned}
$$

Equations (34), (36) and (38) of the 3DOF system express the motion in the slip phase in terms of the four coordinates $x_{A}, s_{A}, \delta$ and $\varphi$. Thus, a fourth equation, which captures coupling between $x_{A}$ and $s_{A}$ due to tangential friction contact at point $C$, needs to be established. To this end, Eqs. (24) and (25), where $T$ has been replaced by $T_{\mathrm{f}}$ (Eq. (27)), are combined by eliminating the normal force component $N$, which leads after some algebra to

$$
f_{\mu 1}\left(c_{\mathrm{sh}} \dot{x}_{A}+k_{\mathrm{sh}} x_{A}\right)=f_{\mu 2}\left(c_{\mathrm{sv}} \dot{s}_{A}+k_{\mathrm{sv}} s_{A}\right)
$$

The set of nonlinear ODEs (34), (36), (38) and (39) describes the motion of the interacting roller-soil model in the slip phase. The state space representation of these equations for efficient numerical solution is introduced in Appendix D.

\subsection{Procedure of solution}

The response is obtained numerically by switching between the set of the first-order ODEs for the stick phase (Eq. (52)) and the set of ODEs for the slip phase (Eq. (55)), basically as described in [13]. In the stick phase, the outcomes of Eq. (52) (i.e., $x_{A}, s_{A}, \delta, \dot{x}_{A}, \dot{s}_{A}, \dot{\delta}$ ) are inserted into the original equations of motion 30,31 and 32 , which are solved for the accelerations $\ddot{x}_{A}, \ddot{s}_{A}$, and $\ddot{\delta}$. Accelerations $\ddot{x}_{A}, \ddot{s}_{A}, \ddot{\delta}, \ddot{\varphi}$ of the slip phase are obtained by rearranging Eqs. (34), (36), (38) and (53), where the outcomes of Eq. (55) (i.e., $x_{A}, s_{A}, \delta, \varphi, \dot{x}_{A}, \dot{s_{A}}$, $\dot{\delta}$, and $\dot{\varphi}$ ) have been inserted. In both phases of the stick-slip motion, the desired acceleration components $\ddot{x}_{M}$ and $\ddot{z}_{M}$ of the drum center $M$ are obtained by the evaluation of Eq. (18). This analysis continues until the steady-state response is reached. 


\section{Computed drum response}

\subsection{Overview}

The subsequent studies are based on the machine properties of an HAMM HD $\mathrm{HA}^{+} 90 \mathrm{VO}$ tandem roller [6] listed in Table 1 because for this device acceleration response data of the drum center $M$ recorded in field tests are available [18]. It is assumed that all considered non-cohesive soil conditions exhibit a Poisson's ratio $v$ of 0.3 and a density $\rho$ of $1900 \mathrm{~kg} / \mathrm{m}^{3}$. The shear modulus $G$ of the soil is varied in steps of $5 \mathrm{MN} / \mathrm{m}^{2}$ between 5 and $70 \mathrm{MN} / \mathrm{m}^{2}$. The soil parameters and the corresponding discrete soil stiffness coefficients $k_{\mathrm{sh}}$ and $k_{\mathrm{sv}}$ (Eq. (42)), and damping parameters $c_{\mathrm{sh}}$ and $c_{\mathrm{Sv}}$ (Eq. (45)) are listed in Table 2. Note that $c_{\mathrm{sh}}$ and $c_{\mathrm{sv}}$ do not change with increasing soil stiffness because Poisson's ratio $v$ and soil density $\rho$ are assumed to be constant, compare with Eq. 45. In the base case, a semicircular shaped settlement trough with radius $R=0.606 \mathrm{~m}$ is assumed, which is slightly larger than the drum radius $r=0.60 \mathrm{~m}$. The corresponding spiral parameters representing a semicircle are $k=0$ and $a=R$.

In the following, the steady-state acceleration components of the drum center $M, \ddot{x}_{M}$ and $\ddot{z}_{M}$, and thereof derived characteristic response quantities are presented and discussed because they serve as basis of a novel CCC methodology [18], as discussed in the introduction.

\subsection{Pure rolling (stick) motion of the drum}

At first, the influence of the geometry of the settlement trough on the predicted accelerations of a drum in pure rolling motion is investigated. That is, it is assumed that the tangential contact force $T$ does not exceed the sliding friction $T_{\mathrm{f}}$ at any time. To this end, a semicircular settlement through $(k=0, a=R=0.603 \mathrm{~m})$ as well as an asymmetric settlement trough with the shape of a logarithmic spiral $(k=0.002, a=0.601 \mathrm{~m})$ are considered. In this study, no driving torque is applied, i.e., $M_{\mathrm{Md}}=0$. As an example, Fig. 7 shows for soil

Table 1 Roller parameters $[6,7,18]$

\begin{tabular}{llll}
\hline Parameter & Symbol & Value & Dimension \\
\hline Radius of the drum & $r$ & 0.6 & $\mathrm{~m}$ \\
Width of the drum & $b$ & 1.68 & $\mathrm{~m}$ \\
Mass of the drum & $m$ & 1851 & $\mathrm{~kg}$ \\
Mass moment of inertia of the drum & $I$ & 411.78 & $\mathrm{~kg} \mathrm{~m}$ \\
Static axle load & $P_{0}$ & $\mathrm{~N}$ \\
Static axle load (without drum) & $F_{z}$ & 45,224 & $\mathrm{~N}$ \\
Excitation frequency & $\bar{f}$ & 27,066 & $\mathrm{~Hz}$ \\
Amplitude of the oscillation moment & $M_{\mathrm{Mu}}^{(0)}$ & 39 & $\mathrm{Nm}$ \\
Suspension drum/frame-stiffness & $k_{\mathrm{d}}$ & 54,947 & $\mathrm{~N} / \mathrm{m}$ \\
Suspension drum/frame-damping & $c_{\mathrm{d}}$ & $4 \times 10^{6}$ & $\mathrm{Ns} / \mathrm{m}$ \\
\hline
\end{tabular}

Table 2 Soil parameters (based on [18])

\begin{tabular}{llllllll}
\hline$G\left(\mathrm{MN} / \mathrm{m}^{2}\right)$ & $\rho\left(\mathrm{kg} / \mathrm{m}^{3}\right)$ & $v$ & $a_{0}(\mathrm{~m})$ & $k_{\mathrm{sh}}(\mathrm{MN} / \mathrm{m})$ & $k_{\mathrm{sv}}(\mathrm{MN} / \mathrm{m})$ & $c_{\mathrm{sh}}(\mathrm{kNs} / \mathrm{m})$ & $c_{\mathrm{sv}}(\mathrm{kNs} / \mathrm{m})$ \\
\hline 5 & 1900 & 0.30 & 0.217 & 11.4 & 16.3 & 141 & 263 \\
10 & 1900 & 0.30 & 0.153 & 19.7 & 29.5 & 141 & 263 \\
15 & 1900 & 0.30 & 0.125 & 27.3 & 42.1 & 141 & 263 \\
20 & 1900 & 0.30 & 0.108 & 34.5 & 54.3 & 141 & 263 \\
25 & 1900 & 0.30 & 0.097 & 41.4 & 66.3 & 141 & 263 \\
30 & 1900 & 0.30 & 0.088 & 48.2 & 78.1 & 141 & 263 \\
35 & 1900 & 0.30 & 0.082 & 54.8 & 89.8 & 141 & 263 \\
40 & 1900 & 0.30 & 0.077 & 61.2 & 101 & 141 & 263 \\
45 & 1900 & 0.30 & 0.072 & 67.6 & 113 & 141 & 263 \\
50 & 1900 & 0.30 & 0.069 & 73.9 & 124 & 141 & 263 \\
55 & 1900 & 0.30 & 0.065 & 80.1 & 136 & 141 & 263 \\
60 & 1900 & 0.30 & 0.063 & 86.2 & 147 & 141 & 263 \\
65 & 1900 & 0.30 & 0.060 & 92.3 & 158 & 141 & 263 \\
70 & 1900 & 0.30 & 0.058 & 98.3 & & & 263 \\
\hline
\end{tabular}



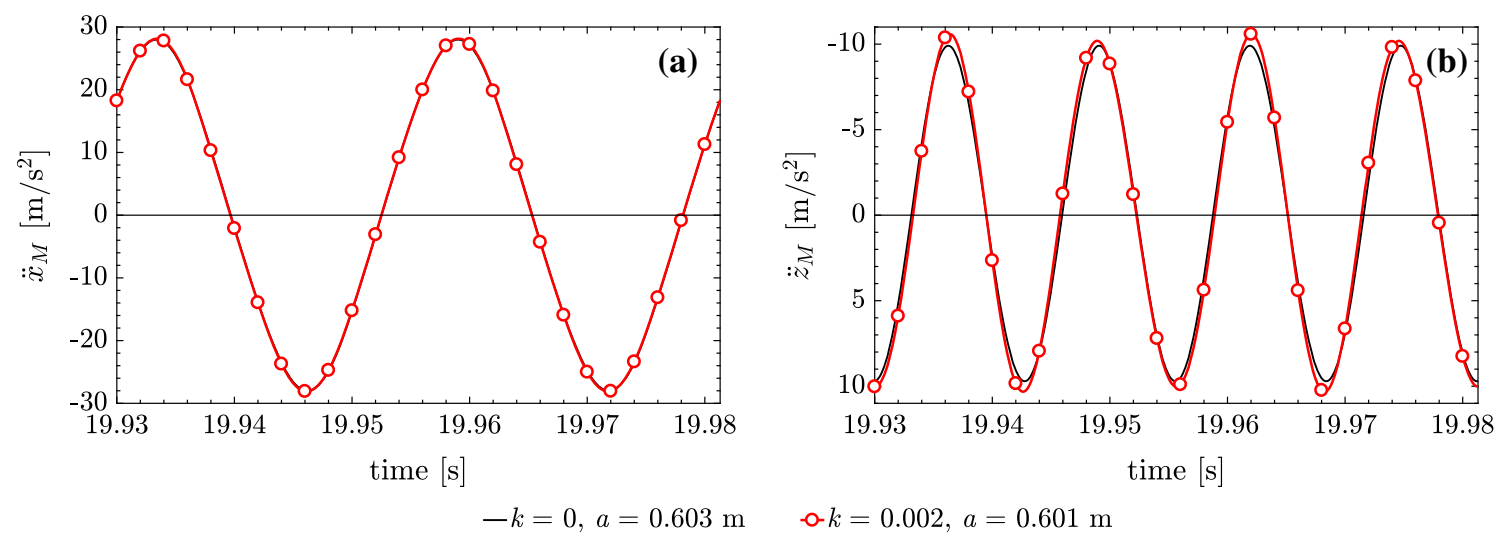

Fig. 7 Time history of the horizontal (a) and vertical (b) accelerations in the drum center; soft soil $G=5 \mathrm{MN} / \mathrm{m}^{2} ; M_{\mathrm{Md}}=0$; pure rolling motion; symmetric versus asymmetric settlement trough

stiffness $G=5 \mathrm{MN} / \mathrm{m}^{2}$ the steady-state acceleration components $\ddot{x}_{M}$ and $\ddot{z}_{M}$ with respect to time $t$ during two excitation periods for both settlement troughs. In Fig. 8a, the time history of the corresponding position angle $\delta$ is displayed, and in Fig. $8 \mathrm{~b} \ddot{z}_{M}$ is plotted against $\ddot{x}_{M}$. The vertical acceleration $\left(\ddot{z}_{M}\right)$ is a result of the upward and downward motions of the drum in the settlement trough during each period of excitation. Due to the nonlinear motion, the period of $\ddot{z}_{M}$ is half of the excitation period, i.e., two excitation periods correspond to four periods of vertical acceleration $\ddot{z}_{M}$. If the settlement trough is of semicircular shape (solid lines in black), both the horizontal and vertical acceleration components of $M$ are symmetric with respect to the horizontal axis. The plot $\ddot{z}_{M}$ versus $\ddot{x}_{M}$, represented in Fig. $8 \mathrm{~b}$ by a solid black line, has the shape of "recumbent eight," and is symmetric with respect to the vertical and horizontal axes. An asymmetric settlement trough results in a slightly asymmetric pattern of the position angle $\delta$ (see Fig. 8a, red line with circular markers), i.e., the amplitudes in the positive domain are larger than those in the negative domain. Consequently, also the peak values of the vertical acceleration $\ddot{z}_{M}$ in the positive domain become slightly larger (see Fig. 7b). The horizontal acceleration $\ddot{x}_{M}$, which has the same period as the excitation period, remains virtually unaffected from the shape of the settlement trough (see Fig. 7a). Thus, the plot $\ddot{x}_{M}-\ddot{z}_{M}$ shown by red lines with circular markers becomes slightly asymmetric, as seen in Fig. $8 \mathrm{~b}$. Since these and further results not presented here have revealed that the asymmetry of the settlement trough has only a very small effect on the response, subsequently, the drum acceleration is examined based on a semicircular settlement trough (i.e., $k=0$ ).

Next, the influence of the radius $R$ of a semicircular settlement trough on the drum center acceleration is investigated for all considered soil shear moduli $G$, for $M_{\mathrm{Md}}=0$. Fig. 9 shows the peak acceleration components, $\ddot{x}_{M \max }$ (a) and $\ddot{z}_{\text {Mmax }}$ (b), respectively, as a function of $G$ for four selected radii $R$ ranging from 0.603 to $0.65 \mathrm{~m}$. As observed, the horizontal peak acceleration $\ddot{x}_{\text {Mmax }}$ increases continuously from about 25-
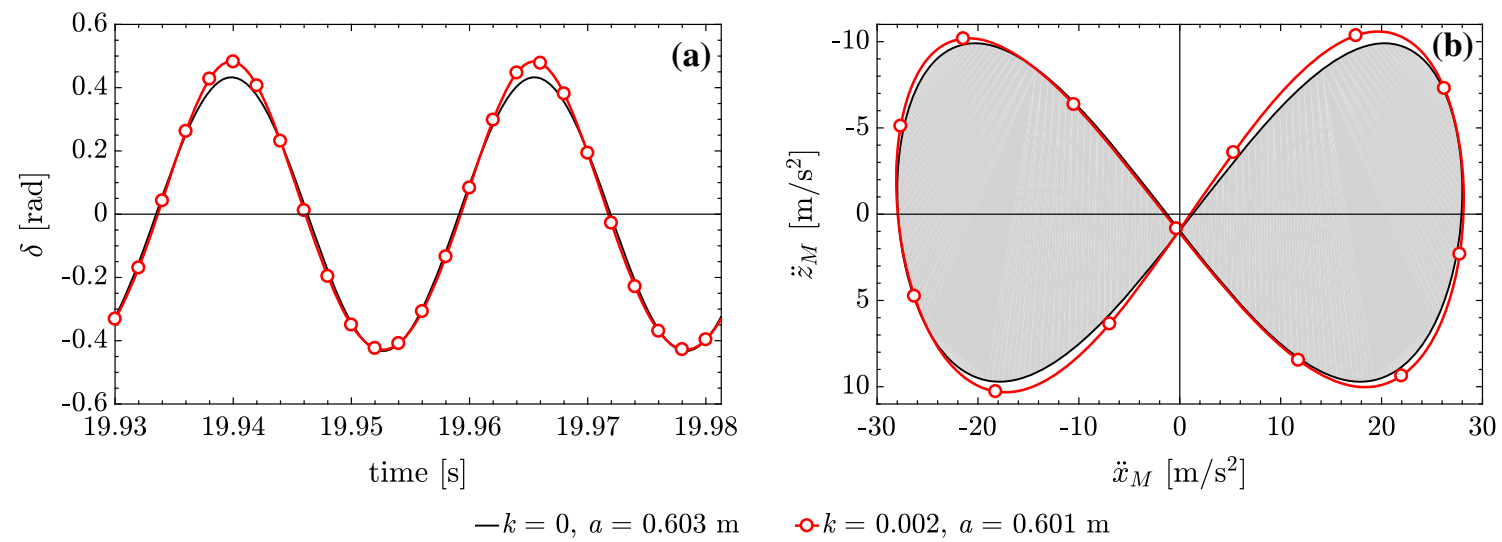

Fig. 8 Time history of position angle $\delta$ (a) and plot $\ddot{x}_{M}$ versus $\ddot{z}_{M}(\mathbf{b})$; soft soil $G=5 \mathrm{MN} / \mathrm{m}^{2} ; M_{\mathrm{Md}}=0$; pure rolling motion; symmetric versus asymmetric settlement trough 

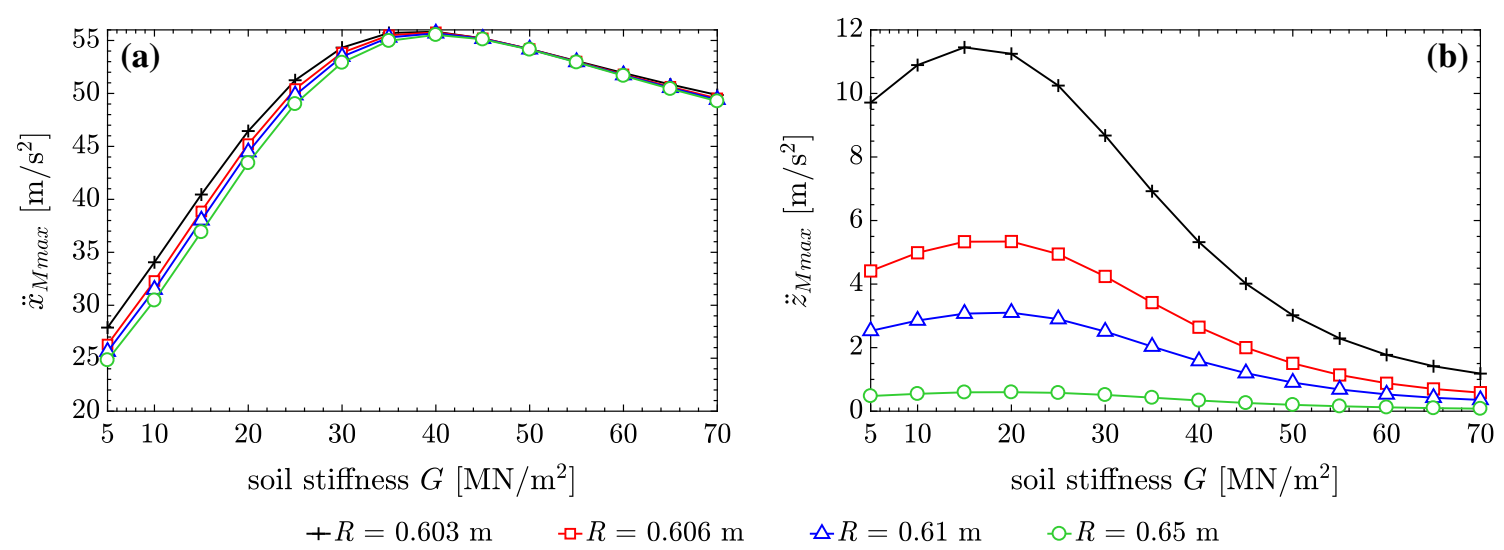

Fig. 9 Peak of the horizontal (a) and vertical (b) accelerations in the drum center as a function of soil shear modulus $G$ for selected radii $R$ of a semicircular settlement trough; $M_{\mathrm{Md}}=0$; pure rolling motion
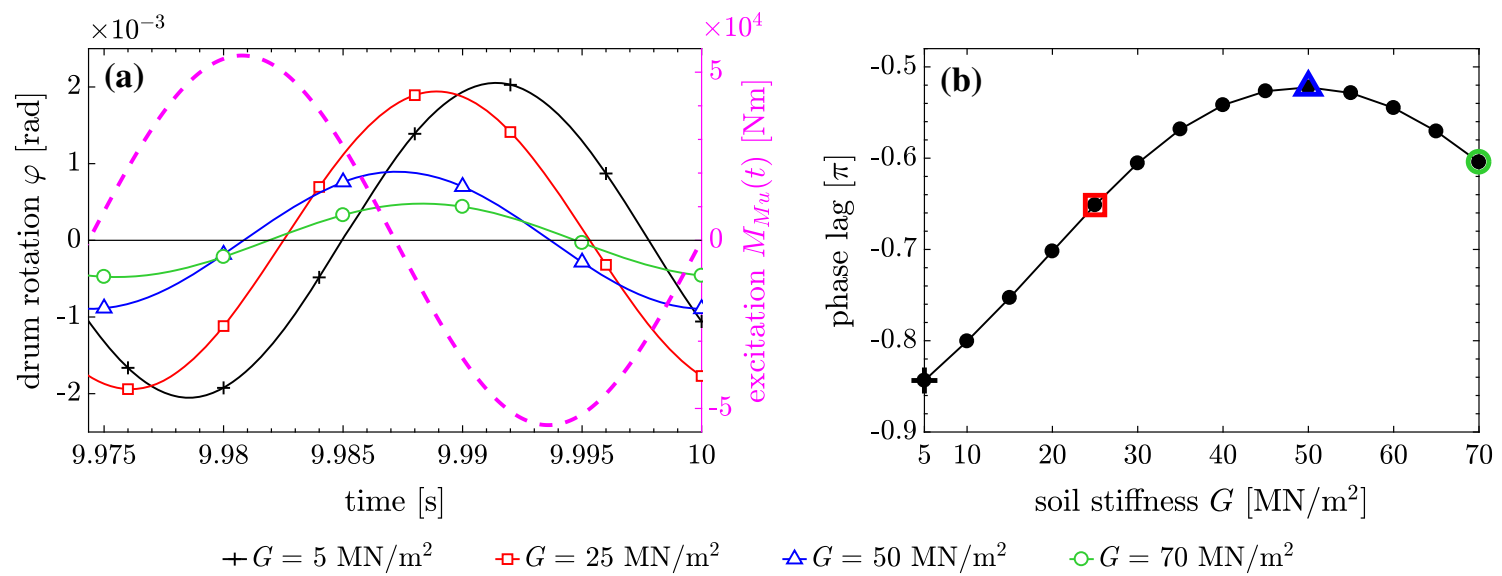

Fig. 10 Phase lag between excitation torque $M_{\mathrm{Mu}}(t)$ (dashed line) and drum rotation $\varphi$-time histories for four selected soil shear moduli $G(\mathbf{a})$, and phase lag for the entire range of $G(\mathbf{b}) ; M_{\mathrm{Md}}=0$; pure rolling motion

$28 \mathrm{~m} / \mathrm{s}^{2}\left(G=5 \mathrm{MN} / \mathrm{m}^{2}\right)$ to about $56 \mathrm{~m} / \mathrm{s}^{2}$ at $G=40 \mathrm{MN} / \mathrm{m}^{2}$, where the maximum is attained, and then slightly descends to $50 \mathrm{~m} / \mathrm{s}^{2}$ at $G=70 \mathrm{MN} / \mathrm{m}^{2}$, almost unaffected by the radius $R$ (Fig. 9a). Vertical accelerations $\ddot{z}_{\text {Mmax }}$ also increase with increasing stiffness $G$; however, they reach their maximum at lower stiffness of $G=15-$ $20 \mathrm{MN} / \mathrm{m}^{2}$, and then, decrease with increasing soil stiffness with a steep gradient (see Fig. $9 \mathrm{~b}$ ). In contrast to $\ddot{x}_{\text {Mmax }}$, the magnitudes of $\ddot{z}_{\text {Mmax }}$ are sensitive to the radius $R$ of the settlement trough, i.e., the closer $R$ is to the drum radius $r$, the larger becomes $\ddot{z}_{\mathrm{Mmax}}$. In general, the vertical peak acceleration components are smaller than the corresponding horizontal ones. As observed, the ratio $\ddot{z}_{\text {Mmax }}$ to $\ddot{x}_{M \max }$ decreases from 0.21 at $R=0.603 \mathrm{~m}$ to about 0.01 at $R=0.65 \mathrm{~m}$. If $R=0.606 \mathrm{~m}, \ddot{z}_{\text {Mmax }}$ is about $10 \%$ of $\ddot{x}_{\text {Mmax }}$. The following studies are based on a settlement trough radius of $R=0.606 \mathrm{~m}$, i.e., $R$ is one percent larger than the drum radius $r$.

Figure 10 illustrates that also the phase lag between the harmonic excitation torque $M_{\mathrm{Mu}}(t)$ and the drum rotation $\varphi\left(=\frac{R-r}{r} \delta\right)$ strongly depends on the soil stiffness $G$. Fig. 10a shows one period of the harmonic excitation torque $M_{\mathrm{Mu}}(t)$ (dashed line) and the corresponding drum rotation $\varphi$ for four values of the soil stiffness $G$, as specified in the legend. In this representation, the effect of $G$ on both the phase lag and the amplitude of $\varphi$ becomes apparent. The maximum of the phase lag occurs at $G=50 \mathrm{MN} / \mathrm{m}^{2}$ (Fig. 10b). At the lowest considered soil stiffness, $G=5 \mathrm{MN} / \mathrm{m}^{2}$, the phase lag is $-0.84 \pi$. For $G \rightarrow 0$ (lifted drum), the phase lag converges toward $-\pi$. That is, an oscillation drum without soil contact rotates purly forward-backward with a phase lag $-\pi$ between $M_{\mathrm{Mu}}(t)$ and $\varphi$.

The steady-state acceleration components $\ddot{x}_{M}$ and $\ddot{z}_{M}$ (see Figs. 11a, b, respectively) of the drum center $M$ demonstrate the effect of the soil stiffness on the peak response and phase lag, exemplarily shown for four selected shear moduli $G$ and two excitation periods. While the maximum of $\ddot{x}_{M}$ is about the same for $G=25$, 50 and $70 \mathrm{MN} / \mathrm{m}^{2}$, the peak value of $\ddot{z}_{M}$ related to $G=25 \mathrm{MN} / \mathrm{m}^{2}$ is about 3.3 (8.4) times larger than for 

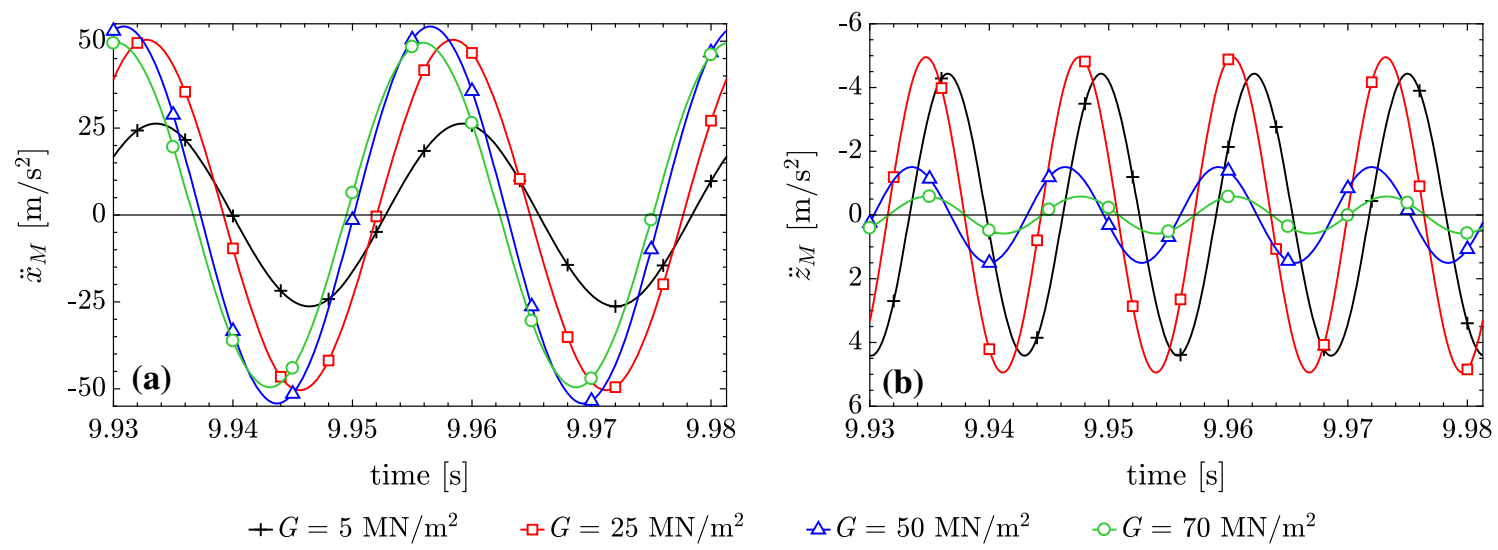

Fig. 11 Steady-state horizontal (a) and vertical (b) accelerations in the drum center for four selected soil shear moduli $G$; $M_{\mathrm{Md}}=0$; pure rolling motion

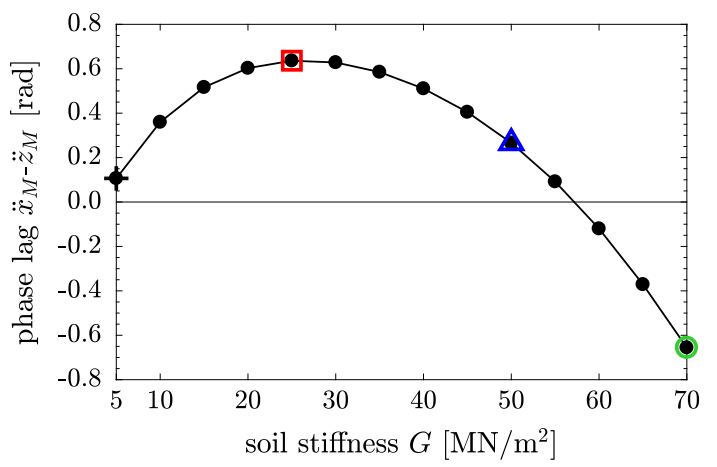

Fig. 12 Phase lag between $\ddot{x}_{M}$ and $\ddot{z}_{M}$ as a function of shear modulus $G ; M_{\mathrm{Md}}=0$; pure rolling motion

$G=50(70) \mathrm{MN} / \mathrm{m}^{2}$. Another observation is that the period of $\ddot{z}_{M}$ is only half of the period of $\ddot{x}_{M}$, which corresponds to the excitation period. Additionally, in Fig. 12 the phase lag between $\ddot{x}_{M}$ and $\ddot{z}_{M}$ is depicted. As observed, the predicted phase lag is about $0.11 \mathrm{rad}$ for the lowest shear modulus, the maximum of 0.64 is obtained at $G=25 \mathrm{MN} / \mathrm{m}^{2}$, and with increasing soil stiffness it subsequently decreases. For stiff soils with $G \geq 60 \mathrm{MN} / \mathrm{m}^{2}$, the phase is negative. Note that a positive phase lag indicates that $\ddot{z}_{M}$ lags behind $\ddot{x}_{M}$. Plotting the vertical component $\ddot{z}_{M}$ against its horizontal counterpart $\ddot{x}_{M}$ results in another meaningful response representation, shown in Fig. 13a. The result is a so-called Lissajous curve [10], whose shape is either similar to the lemniscate of Gerono [12] (resembles an eight curve [12], for $G=5,50$ and $70 \mathrm{MN} / \mathrm{m}^{2}$ ), or similar to a general besace (for $\left.G=25 \mathrm{MN} / \mathrm{m}^{2}\right)$. In the latter case $\left(G=25 \mathrm{MN} / \mathrm{m}^{2}\right)$, the node of the resulting figure is shifted considerably along the vertical axis in the positive domain. This is a result of the phase lag between $\ddot{x}_{M}$ and $\ddot{z}_{M}$, which has its maximum at $G=25 \mathrm{MN} / \mathrm{m}^{2}$ (see Fig. 12). All figures are symmetric with respect to the vertical axis because the driving torque is zero, $M_{\mathrm{Md}}=0$.

The response in the frequency domain for four selected subsoils (Figs. 14, 15) reveals that the frequency content $(f)$ of the vertical accelerations $\ddot{z}_{M}$ is two times the excitation frequency $\bar{f}$ if $M_{\text {Md }}=0$ (Fig. 14b). In Figs. 14 and 15 , the frequency $f$ is normalized with respect to the excitation frequency $\bar{f}$. In contrast, $\ddot{x}_{M}$ is governed by $\bar{f}$, see Fig. 14a. Since the dominating frequency content of $\ddot{z}_{M}$ and $\ddot{x}_{M}$ differs by a factor of two, the $\ddot{z}_{M}-\ddot{x}_{M}$ plot results in the Lissajous curves shown in the previous figure.

When additionally a constant driving torque of $M_{\mathrm{Md}}=0.05 M_{\mathrm{Mu}}^{(0)}$ is applied to the drum, the spectrum of vertical accelerations $\ddot{z}_{M}$ contains now two frequencies, one at $f=2 \bar{f}$ (as for $M_{\mathrm{Md}}=0$ ) and one at $f=\bar{f}$ (see Fig. 15b). The spectral amplitudes of $\ddot{z}_{M}$ are largest for $f / \bar{f}=1$, except for the softest subsoil $\left(G=5 \mathrm{MN} / \mathrm{m}^{2}\right)$. The ratio of the amplitude at $f=2 \bar{f}$ to the amplitude at operating frequency $\bar{f}$ decreases from $1.11\left(G=5 \mathrm{MN} / \mathrm{m}^{2}\right)$ to $0.24\left(G=70 \mathrm{MN} / \mathrm{m}^{2}\right)$. The amplitudes at $f / \bar{f}=2$ are virtually the same as for $M_{\mathrm{Md}}=0$ (compare Fig. 14b with Fig. 15b). The frequency spectrum of $\ddot{x}_{M}$ is, however, virtually not affected by the driving torque $M_{\mathrm{Md}}$ (compare Fig. 14a with Fig. 15a). Thus, the application of the driving torque $M_{\mathrm{Md}}$, 

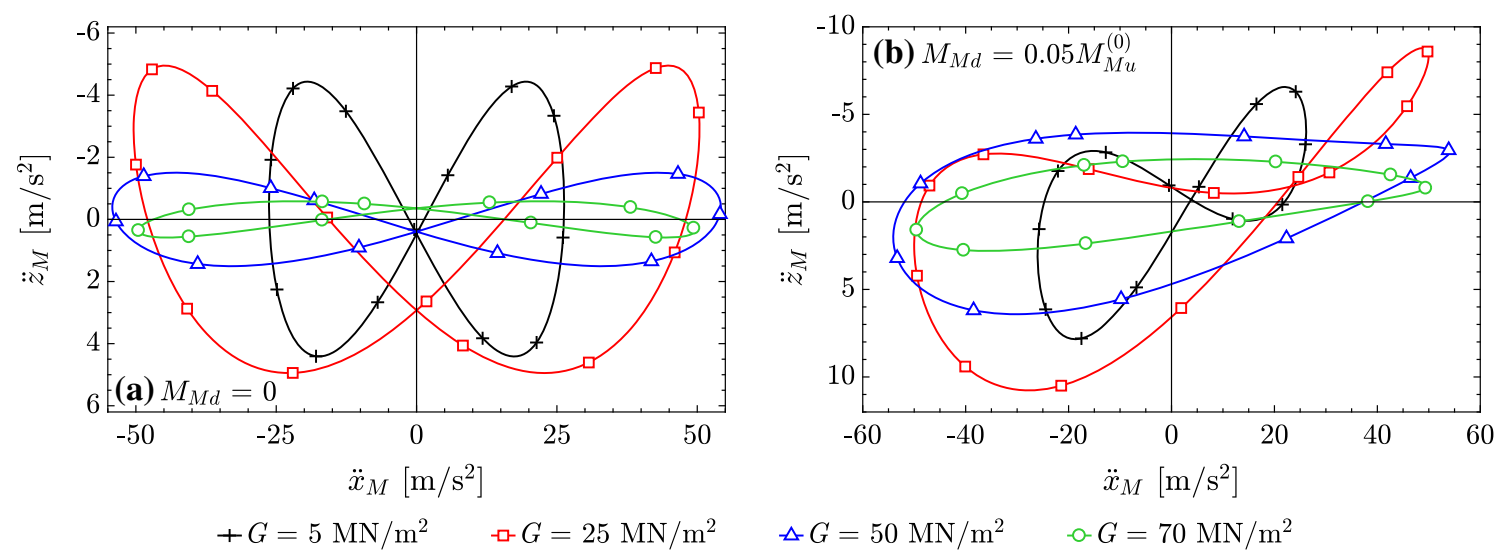

Fig. 13 Plot $\ddot{z}_{M}$ over $\ddot{x}_{M} ; M_{\mathrm{Md}}=0$ (a) versus $M_{\mathrm{Md}}=0.05 M_{\mathrm{Mu}}^{(0)}(\mathbf{b})$; pure rolling motion
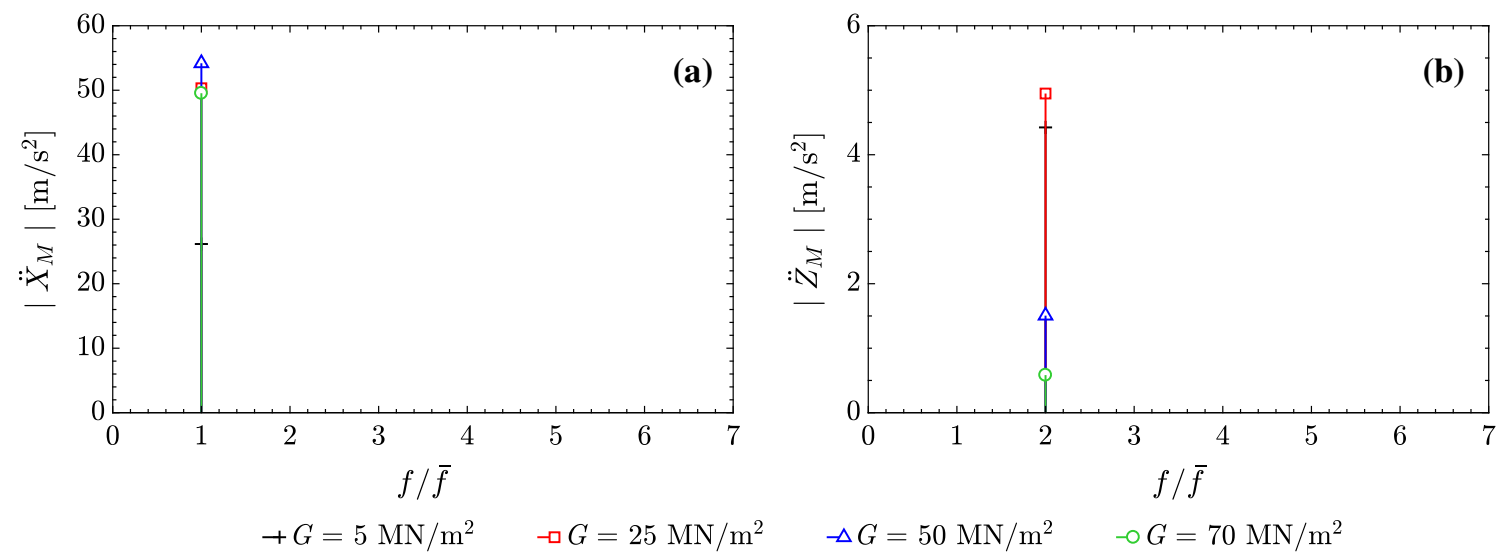

Fig. 14 Frequency spectrum of the horizontal (a) and vertical (b) acceleration in the drum center for four selected shear moduli $G$; pure rolling motion; $M_{\mathrm{Md}}=0$

which captures the effect of the translational drum motion with the roller driving speed, changes the shape of the $\ddot{z}_{M}-\ddot{x}_{M}$ plot, as seen in Fig. 13b. The Lissajous curves become asymmetric and distorted, depending on the soil stiffness and the ratio of the amplitude at $f=2 \bar{f}$ to the amplitude at operating frequency $\bar{f}$. For large soil stiffness, the asymmetric Lissajous similar curves degenerate into curves without node.

Another important response quantity is the area inside the $\ddot{z}_{M}$ over $\ddot{x}_{M}$ figure. Recent experimental studies have revealed that this area increases with increasing shear modulus $G$, and thus, has been proposed as performance indicator of the actual soil compaction [18]. In Fig. 16, this area is plotted against shear modulus $G$, for simulations both without driving torque (black line with "+" markers) and with driving torque (red line with circular markers). It is seen that for zero driving torque, the maximum area is obtained at a quite low stiffness between $G=20 \mathrm{MN} / \mathrm{m}^{2}$ and $G=25 \mathrm{MN} / \mathrm{m}^{2}$. As such, this area would be an appropriate performance indicator only for soil compaction up to soil stiffness of about $G=25 \mathrm{MN} / \mathrm{m}^{2}$. However, in the more realistic modeling scenario, the driving torque $M_{\mathrm{Md}}=0.05 M_{\mathrm{Mu}}^{(0)}$ shifts the maximum of the area to stiffer subsoils with $G=40 \mathrm{MN} / \mathrm{m}^{2}$. Thus, the applicability of the area as compaction control value is extended to a larger soil range.

\subsection{Stick-slip motion of the drum}

The following studies on the stick-slip motion are based on a coefficient of friction of $\mu=0.5$ between drum and soil.

Figures 17 and 18 show for two excitation periods the steady-state time histories of $\ddot{x}_{M}$ and $\ddot{z}_{M}$ for four soil stiffness parameters $G$ specified in the legend, and an excitation torque of $M_{\mathrm{Md}}=0$ (Fig. 17) and 

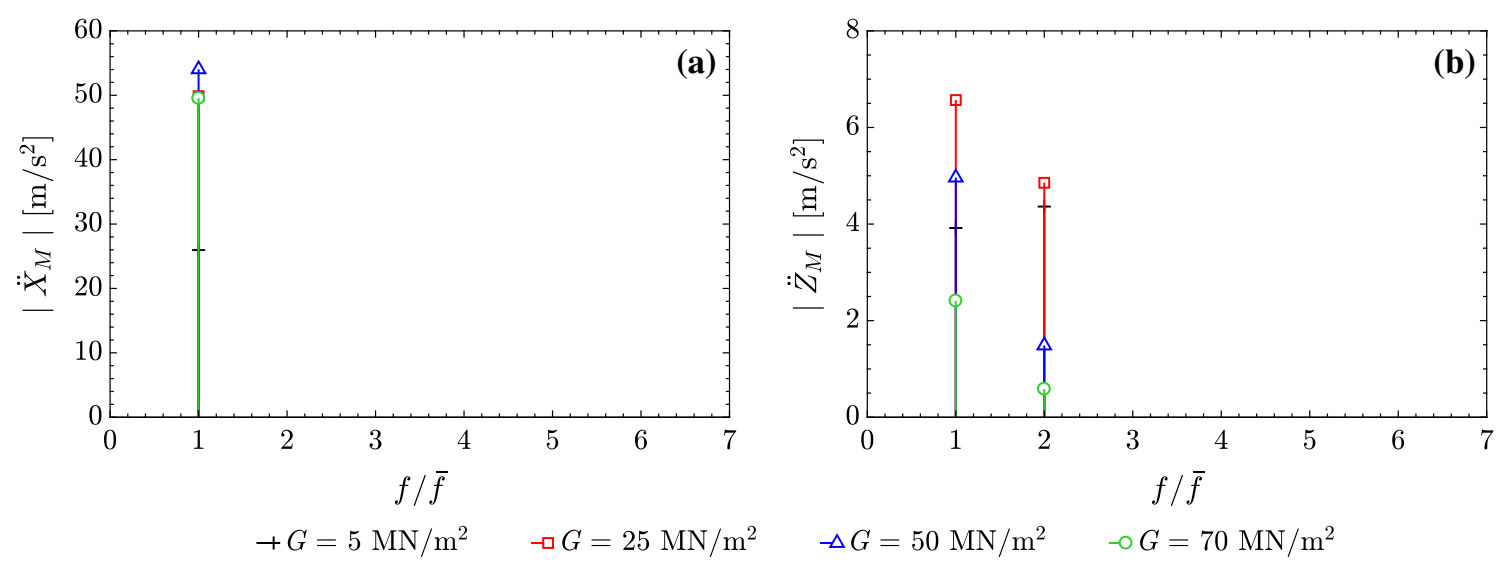

Fig. 15 Frequency spectrum of the horizontal (a) and vertical (b) acceleration in the drum center for four selected shear moduli $G$; pure rolling motion; $M_{\mathrm{Md}}=0.05 M_{\mathrm{Mu}}^{(0)}$

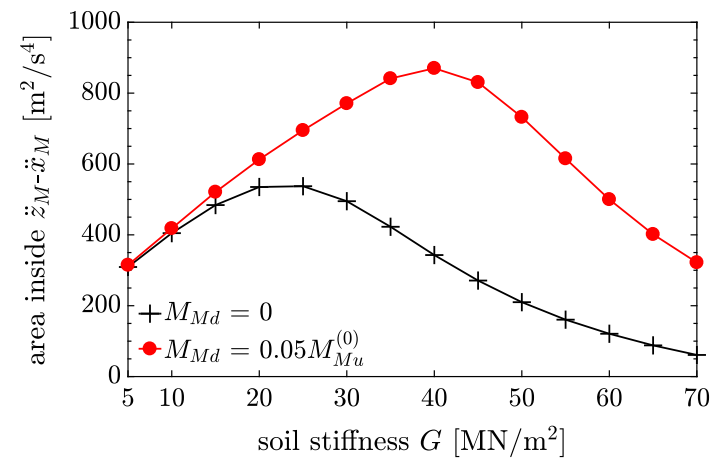

Fig. 16 Area inside the $\ddot{z}_{M}-\ddot{x}_{M}$ figure as a function of the soil shear modulus $G$; pure rolling motion; $M_{\mathrm{Md}}=0$ and $M_{\mathrm{Md}}=$ $0.05 M_{\mathrm{Mu}}^{(0)}$, respectively

$M_{\mathrm{Md}}=0.05 M_{\mathrm{Mu}}^{(0)}$ (Fig. 18), respectively. These results illustrate the grave effect of slip between drum and soil surface on the response. In general, in stick-slip motion the amplitudes of $\ddot{x}_{M}$ and $\ddot{z}_{M}$ are significantly smaller compared to pure rolling of the drum, compare Fig. 17 with Fig. 11. In the slip phase, both the positive and negative peaks of the horizontal drum center acceleration are cut (see Fig. 17a). A kink in $\ddot{x}_{M}$ indicates the transition from the stick to the slip phase and vice versa. During the slip phase, both the horizontal and vertical acceleration components in the drum center decrease. Figure 17a also shows that the slope of $\ddot{x}_{M}$ in the slip phase is larger the lower the soil stiffness. In contrast to $\ddot{x}_{M}$, the component $\ddot{z}_{M}$ decreases significantly with increasing soil shear modulus (see Fig. 17b). The horizontal response without excitation torque (i.e., $M_{\mathrm{Md}}=0$ ) is almost symmetric with respect to the time axis, while for an excitation torque of $M_{\mathrm{Md}}=0.05 M_{\mathrm{Mu}}^{(0)}$ the slip phase in the negative horizontal acceleration range is only about the half of the one in the positive range (compare Fig. 17a with Fig. 18a). Also, the negative response amplitude is larger than the positive one. Another observation is that horizontal steady-state stick-slip acceleration $\ddot{x}_{M}$ is only slightly affected by the soil stiffness parameter, and concerns the duration of the slip phase, which increases slightly with increasing soil stiffness. In contrast, both signature and amplitude of the vertical component $\ddot{z}_{M}$ depend strongly on the subsoil properties (see Fig. 18b). The acceleration components $\ddot{z}_{M}$ look differently in the positive and negative ranges, both for $M_{\mathrm{Md}}=0$ and $M_{\mathrm{Md}}=0.05 M_{\mathrm{Mu}}^{(0)}$. For $M_{\mathrm{Md}}=0$, the lowest shear modulus $G=5 \mathrm{MN} / \mathrm{m}^{2}$ yields the largest vertical acceleration amplitude (see Fig. 17b). Applying a driving torque of $M_{\mathrm{Md}}=0.05 M_{\mathrm{Mu}}^{(0)}$ yields the positive amplitudes of $\ddot{z}_{M}$ between 2 (for $G=5 \mathrm{MN} / \mathrm{m}^{2}$ ) and 7.6 (for $G=70 \mathrm{MN} / \mathrm{m}^{2}$ ) times larger than for $M_{\mathrm{Md}}=0$ (see Fig. 18b). In the negative range, no characteristic response pattern is observed, i.e., the pattern depends strongly on the soil shear modulus $G$.

Figures 19 and 20 represent $\ddot{x}_{M}$ (a) and $\ddot{z}_{M}$ (b) in the frequency domain both for $M_{\mathrm{Md}}=0$ (Fig. 19) and $M_{\mathrm{Md}}=0.05 M_{\mathrm{Mu}}^{(0)}$ (Fig. 20). First, the spectral response without driving torque $\left(M_{\mathrm{Md}}=0\right)$ is discussed. 

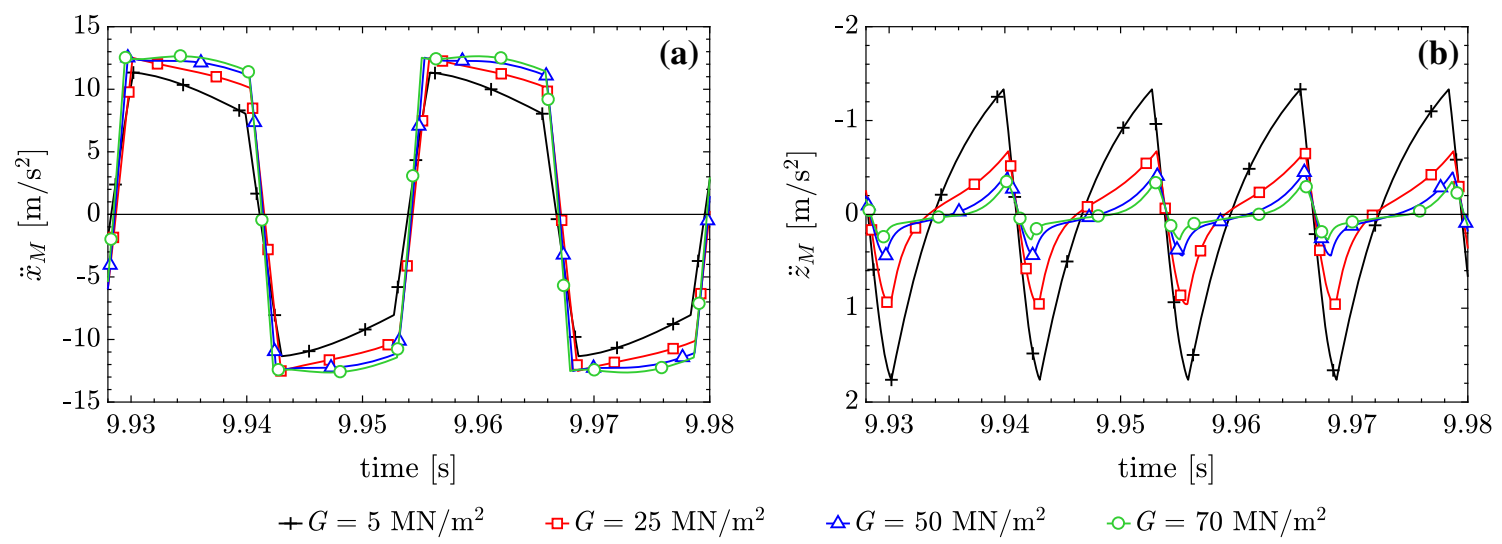

Fig. 17 Steady-state horizontal (a) and vertical (b) accelerations in the drum center for four selected soil shear moduli $G$; $M_{\mathrm{Md}}=0$; stick-slip motion $(\mu=0.5)$
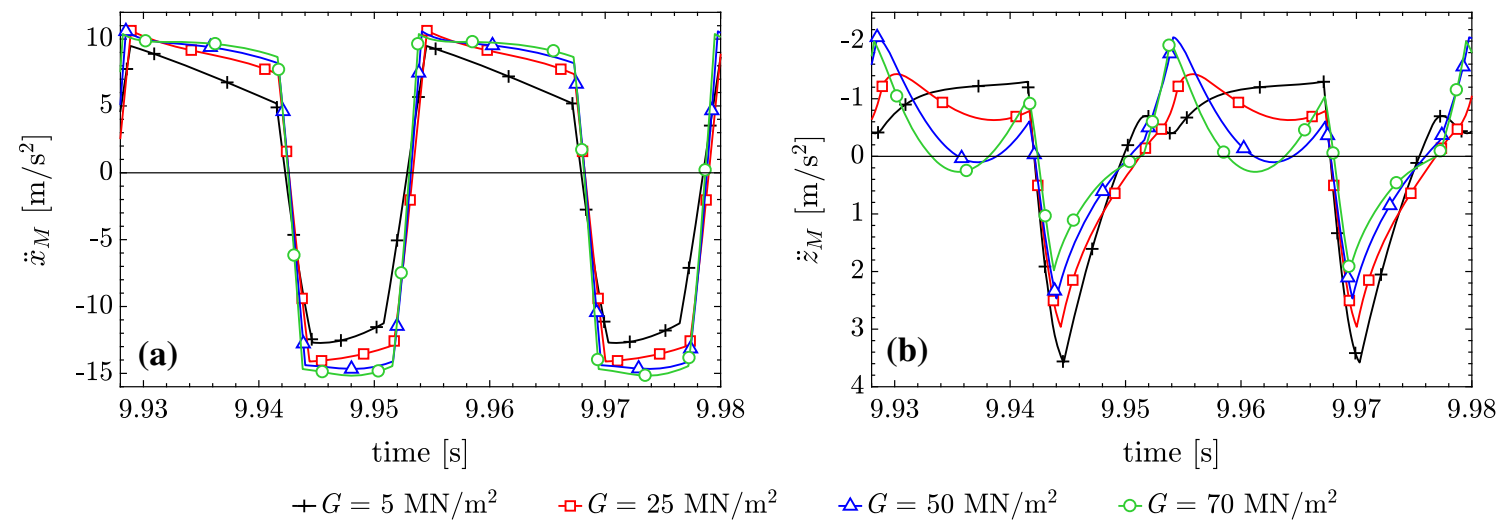

Fig. 18 Steady-state horizontal (a) and vertical (b) accelerations in the drum center for four selected soil shear moduli $M_{\mathrm{Md}}=$ $0.05 M_{\mathrm{Mu}}^{(0)}$ (right); stick-slip motion $(\mu=0.5)$

Fig. 19a, representing the horizontal drum accelerations $\ddot{x}_{M}$, contains only odd harmonics because in the slip phase the response is cut symmetrically. The overtones at uneven multiples of the excitation frequency $(f / \bar{f}=3,5,7, \ldots)$ indicate that the drum motion includes slip phases, compare with the spectrum for pure rolling motion shown in Fig. 14a. The largest amplitude belongs to the operating frequency $f($ i.e., $f / \bar{f}=1$ ). The spectrum of $\ddot{z}_{M}$ for $M_{\text {Md }}=0$ (Fig. 19b) contains only even harmonics $(f / \vec{f}=2,4,6, \ldots)$, with the largest amplitude at $f=2 \bar{f}$. With increasing soil stiffness the higher harmonics in the response become more pronounced, both for $\ddot{x}_{M}$ and $\ddot{z}_{M}$. While with increasing $G$ the amplitudes in the frequency spectrum of $\ddot{x}_{M}$ increase, the amplitudes in the frequency spectrum of $\ddot{z}_{M}$ decrease.

When applying a driving torque of $M_{\mathrm{Md}}=0.05 M_{\mathrm{Mu}}^{(0)}$ to the drum, the spectra for $\ddot{x}_{M}$ and $\ddot{z}_{M}$ contain both odd and even harmonics (see Fig. 20). They are both dominated by the operating frequency $\bar{f}$, except for the stiffest subsoil $G=70 \mathrm{MN} / \mathrm{m}^{2}$. The additional uneven harmonics in the spectrum of $\ddot{z}_{M}(f / \bar{f}=1,3,5, \ldots)$ result from the response asymmetry due to driving torque $M_{\mathrm{Md}}$, whereas the new even harmonics $(f / \bar{f}=4$, 6,8 , etc.) originate from the slip phase of the motion (see Fig. 20b). In the same spectrum, the ratio of the amplitude at $f=2 \bar{f}$ to the amplitude at operating frequency $\bar{f}$ decreases from $0.65\left(G=5 \mathrm{MN} / \mathrm{m}^{2}\right)$ to 0.38 $\left(G=25 \mathrm{MN} / \mathrm{m}^{2}\right)$ and then increases up to about $1.03\left(G=70 \mathrm{MN} / \mathrm{m}^{2}\right)$.

Comparing the $\ddot{z}_{M}-\ddot{x}_{M}$ plot for stick-slip motion and zero driving torque (Fig. 21a) with the corresponding plot for pure rolling motion (Fig. 13a) shows that the slip phase causes a "peak cut" and distortion of the Lissajous similar curves; however, the symmetry with respect to the vertical axis is preserved. The resulting shape is similar to a bow tie (see Fig. 21a). The effect of a driving torque of $M_{\mathrm{Md}}=0.05 M_{\mathrm{Mu}}^{(0)}$ on the $\ddot{z}_{M}-\ddot{x}_{M}$ plot is visualized in Fig. 21b. The resulting curves are twisted, and the symmetry gets lost. Moreover, with increasing soil stiffness the node in the curve moves to the right, and disappears completely for $G=50$ and $70 \mathrm{MN} / \mathrm{m}^{2}$. This behavior has already been observed in pure rolling motion, as shown in Fig. 13b. 

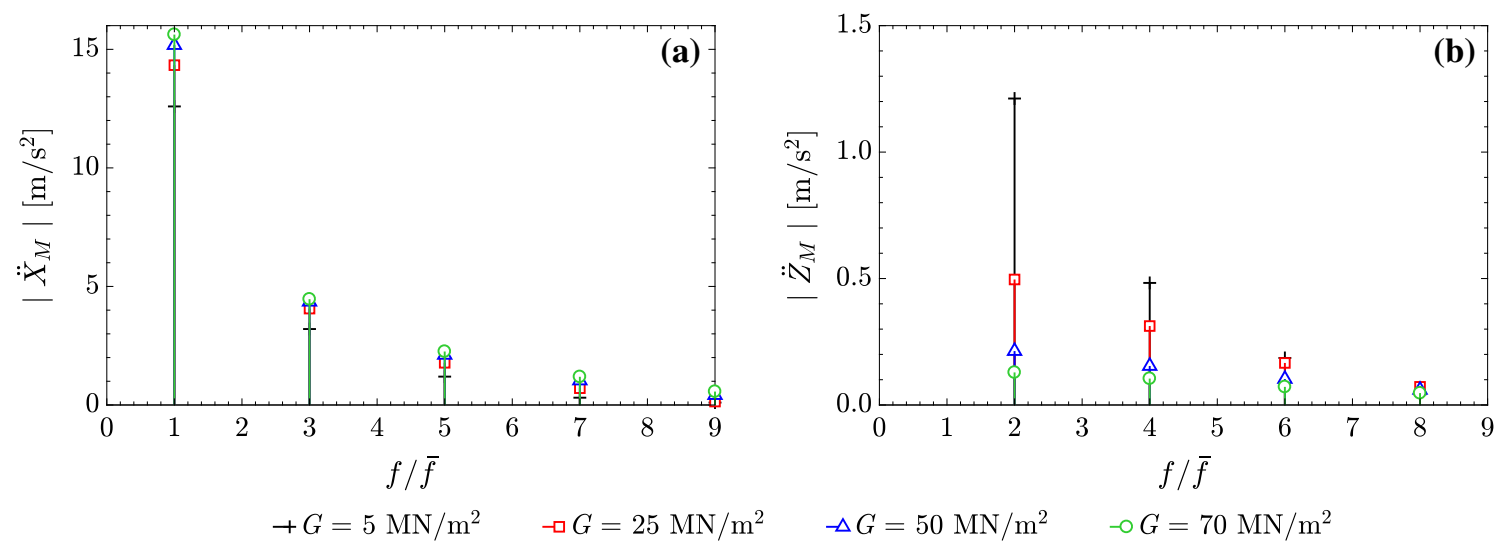

Fig. 19 Frequency spectrum of the horizontal (a) and vertical (b) accelerations in the drum center for four selected shear moduli $G$; stick-slip motion $(\mu=0.5) ; M_{\mathrm{Md}}=0$
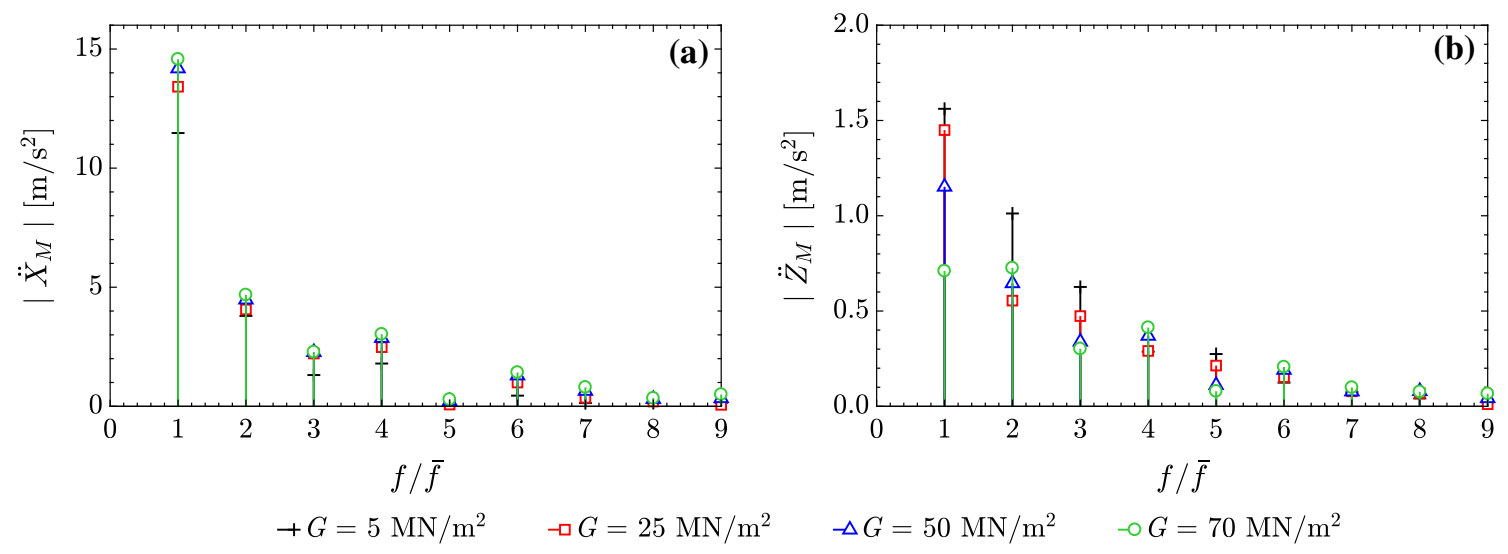

Fig. 20 Frequency spectrum of the horizontal (a) and vertical (b) accelerations in the drum center for four selected shear moduli $G$; stick-slip motion $(\mu=0.5) ; M_{\mathrm{Md}}=0.05 M_{\mathrm{Mu}}^{(0)}$
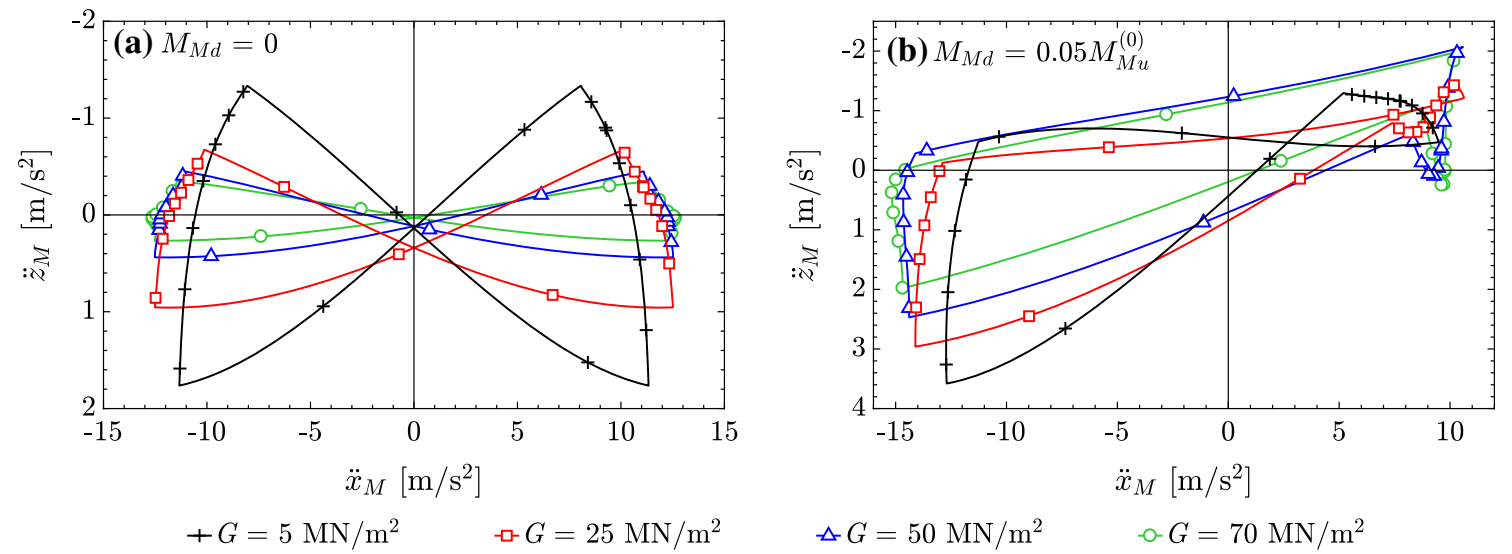

Fig. 21 Vertical $\left(\ddot{z}_{M}\right)$ over horizontal $\left(\ddot{x}_{M}\right)$ acceleration in the drum center; stick-slip motion; $M_{\mathrm{Md}}=0$ (left) and $M_{\mathrm{Md}}=$ $0.05 M_{\mathrm{Mu}}^{(0)}$ (right) 


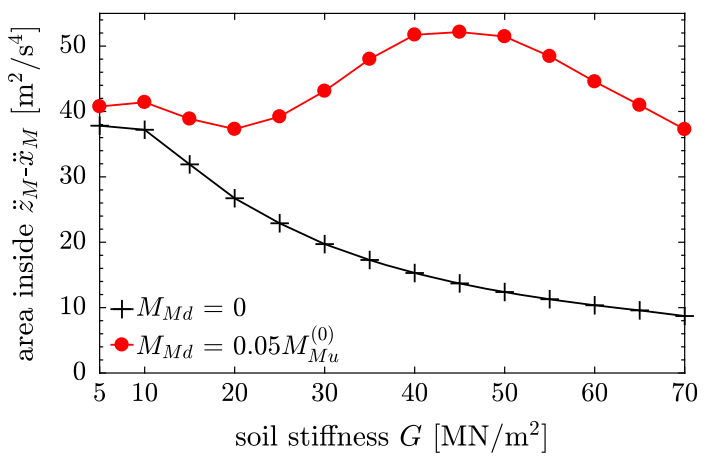

Fig. 22 Area inside the $\ddot{z}_{M}-\ddot{x}_{M}$ figure as a function of the soil shear modulus $G$; stick slip motion; $M_{\mathrm{Md}}=0$ and $M_{\mathrm{Md}}=0.05 M_{\mathrm{Mu}}^{(0)}$, respectively
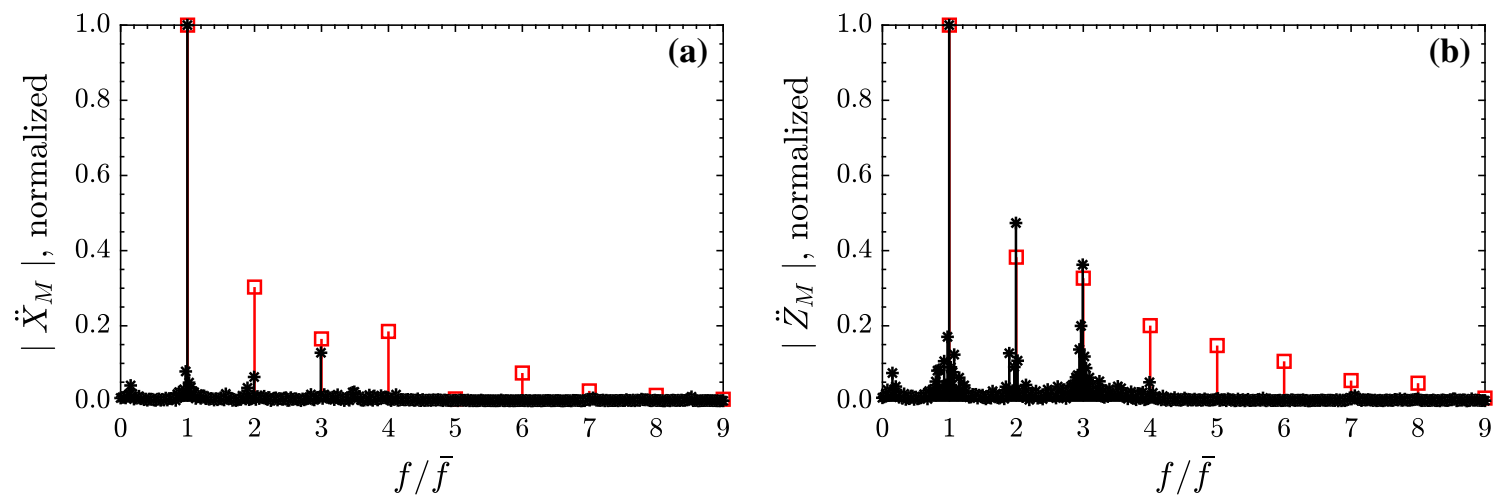

$\neg$ computed $\left(G=25 \mathrm{MN} / \mathrm{m}^{2}\right) \quad *$ recorded (in situ tests, dense gravel)

Fig. 23 Frequency spectrum of the horizontal (a) and vertical (b) accelerations in the drum center; computed $\left(G=25 \mathrm{MN} / \mathrm{m}^{2}\right.$; $\mu=0.5 ; M_{\mathrm{Md}}=0.05 M_{\mathrm{Mu}}^{(0)}$ ) and recorded (in field tests, dense gravel, based on [18]) accelerations

Since in the stick-slip motion the amplitudes of the drum accelerations are smaller than those for the pure rolling motion, the area inside the $\ddot{z}_{M}-\ddot{x}_{M}$ curves decreases. In Fig. 22, this area is depicted as a function of the underlying soil shear stiffness $G$. For the case that the drum oscillates at the bottom of the settlement trough $\left(M_{\mathrm{Md}}=0\right)$, this area shown by a black line with "+" markers decreases continuously with increasing soil stiffness (see Fig. 22). This result is in contradiction to outcomes of field tests presented in [18]. A driving torque of $M_{\mathrm{Md}}=0.05 M_{\mathrm{Mu}}^{(0)}$ increases the area in the whole considered soil parameter range compared to $M_{\mathrm{Md}}=0$, as seen in this figure. The red line with circular markers representing this area exhibits a minimum at $G=20 \mathrm{MN} / \mathrm{m}^{2}$ and a maximum at $G=45 \mathrm{MN} / \mathrm{m}^{2}$. For pure rolling motion, the maximum occurs at slightly smaller soil stiffness of $G=40 \mathrm{MN} / \mathrm{m}^{2}$ (see Fig. 16). However, the minimum only appears in the stick-slip motion.

To support the proposed analytical model, the frequency content of the computed results and of selected drum accelerations recorded during in situ field tests [18] is examined. Figure 23 shows frequency spectra of $\ddot{x}_{M}$ (a) and $\ddot{z}_{M}$ (b) for a dense gravel, i.e., $G=25 \mathrm{MN} / \mathrm{m}^{2}$. As observed, up to the third harmonic the computed and the recorded responses are in good agreement. In the horizontal accelerations, the overtone at the second multiple of the excitation frequency is overestimated by the analytical model (see Fig. 23a). In the frequency range $f / \bar{f}>3$, the normalized amplitudes of the computed accelerations are larger than the ones of the measured response. This is due to the fact that in the measured response, the transition from the stick to slip phases is smoother than that in the analytical model; consequently, the "peak cut" of the horizontal drum accelerations is less pronounced. One reason is the coefficient of friction between the drum and subsoil, which is assumed to be constant in the analytical model. In the present paper, a constant value of $\mu=0.5$ has been assumed, in reality, however, this parameter varies within a certain range. Nevertheless, the analytical model is capable of predicting the overall response behavior observed in the field. 


\section{Conclusions}

For an oscillation roller interacting with the subsurface during soil compaction, a lumped parameter model has been proposed. The governing highly nonlinear equations of this model with three degrees-of-freedom have been derived separately for the stick and the slip phases of the stick-slip motion of the drum.

The presented results demonstrate that the proposed model predicts the main outcomes found in previous in situ tests. The maximum amplitude in the frequency spectrum of the horizontal drum acceleration $\ddot{x}_{M}$ occurs at the operating frequency $\bar{f}$. The overtones at odd multiples of the excitation frequency $(f / \bar{f}=3,5,7, \ldots)$ result from the slip motion phase. In the frequency spectrum of vertical drum accelerations $\ddot{z}_{M}$, the harmonics at even multiples of the excitation frequency $(f / \vec{f}=4,6,8, \ldots)$ indicate slip between the drum and soil. Without driving torque, the maximum amplitude in the frequency spectrum of $\ddot{z}_{M}$ occurs at a frequency equal to $2 \bar{f}$. If the drum operates on the slope of the settlement trough (above its bottom) by the application of a constant driving torque, for most subsoil conditions the dominating frequency corresponds to excitation frequency $\bar{f}$.

It has been confirmed that the function $\ddot{z}_{M}$ against $\ddot{x}_{M}$ shapes a Lissajous similar curve ("recumbent eight"), and the area enclosed in the $\ddot{z}_{M}-\ddot{x}_{M}$ plot depends on the soil stiffness (and thus, on the compaction degree) if the drum conducts a pure rolling motion. Application of a constant driving torque, which simulates the effect of the translation motion of the roller speed, yields a distorted and rotated "recumbent eight," as it is observed from accelerations recorded in field tests. In the stick-slip drum motion, the $\ddot{z}_{M}-\ddot{x}_{M}$ figure becomes cut (shape of a twisted bow tie) because the maximum horizontal accelerations are confined in the slip phase. The found results indicate that relations between the compaction degree of soil layers and the area inside the $\ddot{x}_{M}-\ddot{z}_{M}$ plot can be established also for stick-slip motion of the drum, thus, supporting a recently proposed performance indicator for continuous compaction control for oscillation rollers.

The results of this study are based on the parameters of one specific oscillation roller. Thus, additional simulations using the device parameters of different rollers are required to verify the findings. In a comprehensive parametric study, the excitation frequency and the properties of the rubber buffers should be varied as well. Furthermore, sensitivity studies on the effect of the coefficient friction on the drum acceleration, and consequently, on the area enclosed in the function $\ddot{z}_{M}$ against $\ddot{x}_{M}$ need to be conducted.

Acknowledgements Open access funding provided by University of Innsbruck and Medical University of Innsbruck. The financial support granted by the manufacturer of compaction equipment HAMM AG (Grant No. P8440-022-028) makes the ongoing research on oscillation rollers possible and is gratefully acknowledged.

Open Access This article is distributed under the terms of the Creative Commons Attribution 4.0 International License (http:// creativecommons.org/licenses/by/4.0/), which permits unrestricted use, distribution, and reproduction in any medium, provided you give appropriate credit to the original author(s) and the source, provide a link to the Creative Commons license, and indicate if changes were made.

\section{Appendix A: Dynamic decoupling of drum and roller frame}

For the considered HAMM HD ${ }^{+} 90 \mathrm{VO}$ tandem roller [6], the dynamic decoupling of drum and roller frame, realized by rubber buffers (suspension) mounted between drum and front frame, is analyzed using a simplified model. The suspension is idealized as a Kelvin-Voigt model consisting of a spring and dashpot damper (stiffness $k_{\mathrm{d}}$ and damping parameter $c_{\mathrm{d}}$, respectively), as shown in Fig. 24. The frame (including the cabin) is modeled as a lumped mass, yielding in combination with the Kelvin-Voigt suspension model an SDOF system. The lumped mass $m_{\mathrm{f}}(=2760 \mathrm{~kg})$ identified from the static axle load $P_{0}$, represents the effective mass of the roller with respect to the front axle. The absolute displacement transmissibility $T_{\mathrm{p}}$ of this SDOF system is assessed according to the subsequent well-known equation [4]:

$$
T_{\mathrm{p}}=\sqrt{\frac{1+\left(2 \zeta \frac{\bar{v}}{\omega}\right)^{2}}{\left[1-\left(\frac{\bar{v}}{\omega}\right)^{2}\right]^{2}+\left(2 \zeta \frac{\bar{v}}{\omega}\right)^{2}}}, \quad \zeta=\frac{c_{\mathrm{d}}}{2 m_{\mathrm{f}} \omega}, \omega=\sqrt{\frac{k_{\mathrm{d}}}{m_{\mathrm{f}}}} .
$$

For the roller parameters listed in Table 1, the natural circular frequency $\omega$ is $38.1 \mathrm{rad} / \mathrm{s}$, the damping ratio $\zeta$ is $=0.014$, and the excitation frequency $\bar{v}$ of base excitation $z_{M}$ is $245 \mathrm{rad} / \mathrm{s}$. Since the ratio $\bar{v} / \omega=6.4$ is much larger than $\sqrt{2}$, the absolute displacement transmissibility $T_{\mathrm{p}}$ is much less than 1 . In particular, evaluation of 
this equation yields $T_{\mathrm{p}}=0.025$. That is, the amplitude of the frame displacement $z_{\mathrm{f}}$ is only about $2.5 \%$ of the amplitude of the displacement $z_{M}$ imposed at the frame base (according to the model in Fig. 24). Thus, it is confirmed that drum and front frame are dynamically decoupled by the deeply tuned rubber buffers (as desired by the manufacturer and proofed during roller operation on-site).

\section{Appendix B: Coefficients of the discrete soil model}

In the current study, where only non-cohesive soils are considered ( $\left.v \leq \frac{1}{3}\right)$, according to Wolf [25] the elastic continuous soil halfspace can be reduced to two spring-dashpot damper elements in parallel, as shown in Fig. 2. The corresponding dynamic soil spring coefficients, $k_{\mathrm{sh}}$ and $k_{\mathrm{sv}}$, do not depend on the excitation frequency (because $v \leq \frac{1}{3}$ ), and thus, represent the "frequency-independent coefficients of an ordinary spring (the static stiffness)," as discussed in [25]. Also, the soil damping coefficients, $c_{\mathrm{sh}}$ and $c_{\mathrm{sv}}$, do not depend on the excitation frequency, and thus, represent "frequency-independent coefficients of an ordinary dashpot" [25]. According to Wolf [25], both the dynamic stiffness coefficient and the damping coefficient of the translational cone models are "very accurate in the intermediate- and higher-frequency ranges, whereas in the lower-frequency range $\left(\bar{a}_{0}<2\right)$ and for $v \leq \frac{1}{3}[\ldots]$ the spring-damper soil model overestimates (radiation) damping to a certain extent, especially in the vertical motion." Note that $\bar{a}_{0}$ denotes a non-dimensional frequency parameter [25],

$$
\bar{a}_{0}=\frac{\bar{v} r_{0}}{C_{s}}
$$

with the excitation frequency $\bar{v}$, the equivalent radius $r_{0}$, and the shear wave velocity $C_{s}$. In the present case, where an HAMM HD $90 \mathrm{VO}$ tandem roller with an operating frequency of $39 \mathrm{~Hz}$ is considered, the nondimensional frequency $a_{0}$ varies between $0.18\left(G=70 \mathrm{MN} / \mathrm{m}^{2}\right)$ and $1.29\left(G=5 \mathrm{MN} / \mathrm{m}^{2}\right)$, and thus, $a_{0}<2$. The problem is, hence, in the "lower-frequency range," as defined by Wolf [25].

Consequently, the spring coefficients $k_{\mathrm{sh}}$ and $k_{\mathrm{sv}}$ representing the subsoil stiffness can be determined as follows $([17,25])$,

$$
k_{\mathrm{sh}}=\frac{G a_{0}}{2-v}\left[6.8\left(\frac{b_{0}}{a_{0}}\right)^{0.65}+0.8\left(\frac{b_{0}}{a_{0}}\right)+1.6\right], \quad k_{\mathrm{sv}}=\frac{G a_{0}}{1-v}\left[3.1\left(\frac{b_{0}}{a_{0}}\right)^{0.75}+1.6\right],
$$

where $G$ and $v$ are the shear modulus and the Poisson's ratio of the halfspace, respectively, $b_{0}$ denotes the half drum width, $a_{0}$ is the half contact length between drum and soil surface. Contact length $2 a_{0}$ is four times the contact length according to Hertzian theory [20] for non-adhesive elastic contact [18],

$$
2 a_{0}=4\left[2 \sqrt{\frac{2 r P_{0}(1-v)}{\pi G b_{0}}}\right] .
$$

In this equation, $r$ represents the drum radius, and $P_{0}$ denotes the static (vertical) axle load of the roller.

The damping coefficients $c_{\mathrm{sh}}$ and $c_{\mathrm{sv}}$ representing subsoil damping are two times the coefficients proposed by Wolf [25],

$$
c_{\mathrm{sh}}=2 c_{\mathrm{sh}}^{(\text {Wolf })}=2 \rho \sqrt{\frac{G}{\rho}} 4 a_{0} b_{0}, \quad c_{\mathrm{sv}}=2 c_{\mathrm{sv}}^{(\text {Wolf })}=\sqrt{\frac{2(1-v)}{1-2 v}} c_{\mathrm{sh}}
$$

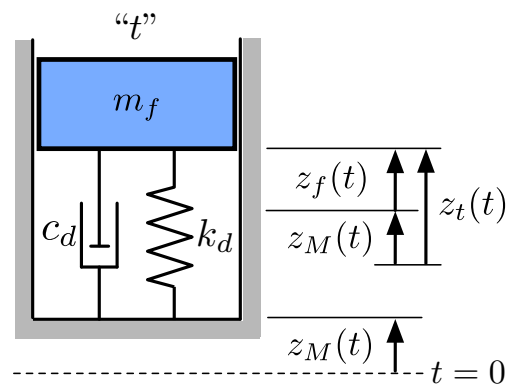

Fig. 24 SDOF model of the front frame supported by the drum suspension 
as discussed in [11]. Variable $\rho$ is the soil density. Substituting for $a_{0}$ the expression of Eq. (43) yields

$$
c_{\mathrm{sh}}=32 \sqrt{\frac{2 \rho r b_{0} P_{0}(1-v)}{\pi}}, \quad c_{\mathrm{Sv}}=64(1-v) \sqrt{\frac{\rho r b_{0} P_{0}}{(1-2 v) \pi}} .
$$

\section{Appendix C: Abbreviations}

The functions $f_{1}, f_{2}, f_{3}, f_{4}, f_{5}, f_{6}$, which depend on the spiral parameters $a, k, \alpha, R_{0}$, drum radius $r$, and angle $\delta(t)$, read as

$$
\begin{aligned}
& f_{1}=R_{0} \exp (-k \delta)[k \cos (\alpha+\delta)+\sin (\alpha+\delta)]-r \cos \delta \\
& f_{2}=R_{0} \exp (-k \delta)\left[\left(1-k^{2}\right) \cos (\alpha+\delta)-2 k \sin (\alpha+\delta)\right]+r \sin \delta, \\
& f_{3}=-R_{0} \exp (-k \delta)[k \sin (\alpha+\delta)-\cos (\alpha+\delta)]+r \sin \delta \\
& f_{4}=-R_{0} \exp (-k \delta)\left[\left(1-k^{2}\right) \sin (\alpha+\delta)+2 k \cos (\alpha+\delta)\right]+r \cos \delta \\
& f_{5}=\sqrt{1+k^{2}} \exp (-k \delta) \frac{R_{0}}{r}-1, \quad f_{6}=-k \sqrt{1+k^{2}} \exp (-k \delta) \frac{R_{0}}{r} .
\end{aligned}
$$

\section{Appendix D: State space representation of the equations of motion}

\section{Stick phase}

The equations of motion for the stick phase, Eqs. (30), (31) and (32), are rewritten in the state space such that

$$
q_{1}=\delta, \quad q_{2}=x_{A}, \quad q_{3}=s_{A}, q_{4}=\dot{\delta}, \quad q_{5}=\dot{x}_{A}, \quad q_{6}=\dot{s}_{A} .
$$

The resulting system of first-order ODEs,

$$
\begin{aligned}
{\left[\begin{array}{cccccc}
1 & 0 & 0 & 0 & 0 & 0 \\
0 & 1 & 0 & 0 & 0 & 0 \\
0 & 0 & 1 & 0 & 0 & 0 \\
0 & 0 & 0 & m f_{1} & m & 0 \\
0 & 0 & 0 & 0 & m \\
0 & 0 & 0 & \left(f_{5} \frac{I}{m r}+\tilde{f}_{1}\right) m & m \cos q_{1} & -m \sin q_{1}
\end{array}\right]\left\{\begin{array}{c}
\dot{q}_{1} \\
\dot{q}_{2} \\
\dot{q}_{3} \\
\dot{q}_{4} \\
\dot{q}_{5} \\
\dot{q}_{6}
\end{array}\right\} } \\
=\left\{\begin{array}{c}
q_{4} \\
q_{5} \\
q_{6} \\
-h_{1}\left(q_{i}(t), q_{i}(t)^{2}\right) \\
m g+F_{z}-h_{2}\left(q_{i}(t), q_{i}(t)^{2}\right) \\
\frac{M_{M}(t)}{r}-\left(m g+F_{z}\right) \sin q_{1}-h_{3}\left(q_{i}(t), q_{i}(t)^{2}\right)
\end{array}\right\}
\end{aligned}
$$

is analyzed numerically by the ode45 solver of MATLAB [16].

\section{Slip phase}

To rewrite the equations of motion for the slip phase, Eqs. (34), (36) and (38), and coupling condition, Eq. (39), in the state space, at first the latter equation needs to be differentiated with respect to time and multiplied by $m / c_{\mathrm{sh}}$,

$$
\begin{aligned}
& f_{\mu 1} m \ddot{x}_{A}-f_{\mu 2} \frac{c_{\mathrm{sv}}}{c_{\mathrm{sh}}} m \ddot{s}_{A}+h_{4}{ }^{(\mathrm{sl})}=0, \\
& h_{4}^{(\mathrm{sl})}=\frac{m}{c_{\mathrm{sh}}}\left\{f_{\mu 1}\left[k_{\mathrm{sh}} \dot{x}_{A}-\left(c_{\mathrm{sv}} \dot{s}_{A}+k_{\mathrm{sv}} s_{A}\right) \dot{\delta}\right]-f_{\mu 2}\left[k_{\mathrm{sv}} \dot{s}_{A}+\left(c_{\mathrm{sh}} \dot{x}_{A}+k_{\mathrm{sh}} x_{A}\right) \dot{\delta}\right]\right\} .
\end{aligned}
$$


The transformations

$$
\begin{aligned}
& q_{1}^{(\mathrm{sl})}=\delta, \quad q_{2}^{(\mathrm{sl})}=x_{A}, \quad q_{3}^{(\mathrm{sl})}=s_{A}, \quad q_{4}^{(\mathrm{sl})}=\varphi, \\
& q_{5}^{(\mathrm{sl})}=\dot{q}_{1}^{(\mathrm{sl})}=\dot{\delta}, \quad q_{6}^{(\mathrm{sl})}=\dot{q}_{2}^{(\mathrm{sl})}=\dot{x}_{A}, \quad q_{7}^{(\mathrm{sl})}=\dot{q}_{3}^{(\mathrm{sl})}=\dot{s}_{A}, \quad q_{8}^{(\mathrm{sl})}=\dot{q}_{4}^{(\mathrm{sl})}=\dot{\varphi}
\end{aligned}
$$

lead to the first-order ODEs for the slip phase,

$$
\begin{aligned}
& {\left[\begin{array}{cccccccc}
1 & 0 & 0 & 0 & 0 & 0 & 0 & 0 \\
0 & 1 & 0 & 0 & 0 & 0 & 0 & 0 \\
0 & 0 & 1 & 0 & 0 & 0 & 0 & 0 \\
0 & 0 & 0 & 1 & 0 & 0 & 0 & 0 \\
0 & 0 & 0 & 0 & m\left(f_{1} f_{\mu 1}-f_{3} f_{\mu 2}\right) & m f_{\mu 1} & -m f_{\mu 2} & 0 \\
0 & 0 & 0 & 0 & m \tilde{f}_{3} & m \sin q_{1}^{\mathrm{sl}} & m \cos q_{1}^{\mathrm{sl}} & 0 \\
0 & 0 & 0 & 0 & 0 & m f_{\mu 1} & -\frac{c_{\mathrm{sv}}}{c_{\mathrm{sh}}} m f_{\mu 2} & 0 \\
0 & 0 & 0 & 0 & \operatorname{sign}\left(v_{\mathrm{rel}}\right) \mu m \tilde{f}_{3} & \operatorname{sign}\left(v_{\mathrm{rel}}\right) \mu m \sin q_{1}^{\mathrm{sl}} & \operatorname{sign}\left(v_{\mathrm{rel}}\right) \mu m \cos q_{1}^{\mathrm{sl}} & \frac{I}{r}
\end{array}\right]\left\{\begin{array}{c}
\dot{q}_{1}^{(\mathrm{sl})} \\
\dot{q}_{2}^{(\mathrm{sl})} \\
\dot{q}_{3}^{(\mathrm{sl})} \\
\dot{q}_{4}^{(\mathrm{sl})} \\
\dot{q}_{5}^{(\mathrm{sl})} \\
\dot{q}_{6}^{(\mathrm{sl})} \\
\dot{q}_{7}^{\text {(sl) }} \\
\dot{q}_{8}^{(\mathrm{sl})}
\end{array}\right\}} \\
& =\left\{\begin{array}{c}
q_{5}^{(\mathrm{sl})} \\
q_{6}^{(\mathrm{sl})} \\
q_{7}^{(\mathrm{sl})} \\
q_{8}^{(\mathrm{sl})} \\
-f_{\mu 2}\left(m g+F_{z}\right)-h_{1}^{(\mathrm{sl})}\left(q_{i}^{(\mathrm{sl})},\left(q_{i}{ }^{(\mathrm{sl})}\right)^{2}\right) \\
\left(m g+F_{z}\right) \cos q_{1}^{\mathrm{sl}}-h_{3}^{(\mathrm{sl})}\left(q_{i}^{(\mathrm{sl})},\left(q_{i}{ }^{(\mathrm{sl})}\right)^{2}\right) \\
-h_{4}^{(\mathrm{sl})}\left(q_{i}^{(\mathrm{sl})},\left(q_{i}^{(\mathrm{sl})}\right)^{2}\right) \\
\frac{M_{M}(t)}{r}-h_{2}^{(\mathrm{sl})}\left(q_{i}{ }^{(\mathrm{sl})},\left(q_{i}{ }^{(\mathrm{sl})}\right)^{2}\right)
\end{array}\right\}
\end{aligned}
$$

which are solved numerically by the ode 45 solver of MATLAB [16].

\section{References}

1. Adam, D.: Continuous Compaction Control (CCC) with Vibrating Rollers. Ph.D. thesis, TU Wien (1996) (in German)

2. Beainy, F., Commuri, S., Zaman, M.: Dynamical response of vibratory rollers during the compaction of asphalt pavements. J. Eng. Mech. 140, 04014039 (2013)

3. Cao, Y.W., Xiang, L., Ma, L.Y., Li, Z.J.: Application analysis of vibrating wheel-soil model based on ABAQUS. In: Advanced Research on Intelligent Systems and Mechanical Engineering, Advanced Materials Research, vol. 644, pp. 366-369. Trans Tech Publications (2013)

4. Clough, R., Penzien, J.: Dynamics of Structures, 2nd edn. McGraw-Hill, New York (1993)

5. Gazetas, G.: Formulas and charts for impedances of surface and embedded foundations. J. Geotech. Eng. 117, 1363-1381 (1991)

6. HAMM AG: Data sheet HD+ 90 VO (2011)

7. HAMM AG: Additional data HD+ 90i (2017)

8. Kearney, E.J.: Oscillatory compaction of hot-mix asphalt. In: Factors Affecting Compaction of Asphalt Pavements, no. E-C105 in Transportation Research Circular, pp. 49-53. Transportation Research Board (2006)

9. Kenneally, B., Musimbi, O.M., Wang, J., Mooney, M.A.: Finite element analysis of vibratory roller response on layered soil systems. Comput. Geotech. 67, 73-82 (2015)

10. Klotter, K.: Vibration Theory. Volume 2: Multi-degrees-of-Freedom Oscillator (in German: Technische Schwingungslehre, 2. Band: Schwinger von mehreren Freiheitsgraden). Springer, Berlin (1981)

11. Kopf, F.: Continuous Compaction Control (CCC) During Compaction of Soil by Means of Dynamic Rollers with Different Kinds of Excitation. Ph.D. thesis, TU Wien (in German) (1999)

12. Lawrence, J.D.: A Catalog of Special Plane Curves. Dover Publications, London (1972)

13. Leine, R., van Campen, D., de Kraker, A., van den Steen, L.: Stick-slip vibrations induced by alternate friction models. Nonlinear Dyn. 16(1), 41-54 (1998)

14. Li, J., Lu, L., Zhou, Z., Xu, L.: Dynamic modeling simulation and analysis of amplitude frequency characteristics on tandem-heavy oscillating rollers. In: IOP Conference Series: Materials Science and Engineering, vol. 382, p. 032040 (2018)

15. Marguerre, K.: Mechanics. 3rd part: Kinetics (in German: Technische Mechanik. Dritter Teil: Kinetik). Heidelberger Taschenbücher. Springer, Berlin (1968)

16. Mathworks: Matlab (version: R2017a) (2017) 
17. Pais, A., Kausel, E.: Approximate formulas for dynamic stiffnesses of rigid foundations. Soil Dyn. Earthq. Eng. 7(4), 213-227 (1988)

18. Pistrol, J.: Compaction with Oscillating Rollers. Motion Behaviour, Roller Integrated Compaction Control and Assessment of Wear. Ph.D. thesis, TU Wien (2016) (in German)

19. Pistrol, J., Adam, D.: Fundamentals of roller integrated compaction control for oscillatory rollers and comparison with conventional testing methods. Transp. Geotech. 17, 75-84 (2018)

20. Popov, V.L.: Contact Mechanics and Friction: Physical Principles and Applications, 2nd edn. Springer, Berlin (2017)

21. Steiner, W.: The use of Castigliano's theorem in Coulomb friction problems. Acta Mech. 225(9), 2471-2483 (2014)

22. Thurner, H., Sandström, Å.: Continuous compaction control, CCC. In: European Workshop Compaction of Soils and Granular Materials, Paris, pp. 237-245 (2000)

23. van Susante, P., Mooney, M.: Capturing nonlinear vibratory roller compactor behavior through lumped parameter modeling. J. Eng. Mech. 134(8), 684-693 (2008)

24. Weisstein, E.W.: Logarithmic spiral (2018). http://mathworld.wolfram.com/Lo-garithmicSpiral.html

25. Wolf, J.: Foundation Vibration Analysis Using Simple Physical Models. Prentice-Hall, Englewood Cliffs (1994)

26. Ziegler, F.: Mechanics of Solids and Fluids, 2nd, corrected 2nd printing edn. Springer, Berlin (1998)

27. Zuwang, S., Shirley, W., Que, W.: The dynamic process of oscillatory compaction and its computer simulation. In: Asia-Pacific Vibration Conference, pp. 532-537. The Korean Society of Mechanical Engineers (1997)

Publisher's Note Springer Nature remains neutral with regard to jurisdictional claims in published maps and institutional affiliations. 